\title{
Exact solution and thermodynamics of a spin chain with long-range elliptic interactions
}

\author{
Federico Finkel and Artemio González-López \\ Departamento de Física Teórica II, Universidad Complutense de Madrid, 28040 \\ Madrid, Spain \\ E-mail: ffinkel@ucm.es and artemio@ucm.es
}

\begin{abstract}
We solve in closed form the simplest $(\mathrm{su}(1 \mid 1))$ supersymmetric version of Inozemtsev's elliptic spin chain, as well as its infinite (hyperbolic) counterpart. The solution relies on the equivalence of these models to a system of free spinless fermions, and on the exact computation of the Fourier transform of the resulting elliptic hopping amplitude. We also compute the thermodynamic functions of the finite (elliptic) chain and their low temperature limit, and show that the energy levels become normally distributed in the thermodynamic limit. Our results indicate that at low temperatures the su(1|1) elliptic chain behaves as a critical $X X$ model, and deviates in an essential way from the Haldane-Shastry chain.
\end{abstract}

Keywords: integrable spin chains (vertex models), solvable lattice models

PACS numbers: 75.10.Pq, 02.30.Ik, 05.30.-d

Submitted to: J. Stat. Mech. 


\section{Introduction}

The spin chain introduced independently by Haldane and Shastry in 1988 is one of the most widely studied integrable lattice models with long-ranged interactions $[1,2]$. This chain describes $N$ equidistant spins on a circle, with two-body interactions inversely proportional to the square of the distance measured along the chord. The Hamiltonian of the Haldane-Shastry (HS) chain can be written as

$$
H=\frac{J \pi^{2}}{N^{2}} \sum_{i<j} \sin ^{-2}\left(\frac{\pi}{N}(i-j)\right)\left(1-S_{i j}\right),
$$

where $S_{i j}$ is the operator permuting the $i$-th and $j$-th spins, and the indices in the double sum range from 1 to $N$. If the Hilbert space of each spin is $m$-dimensional, the operator $S_{i j}$ can be easily expressed in terms of the $\operatorname{su}(m)$ spin operators of the $i$-th and $j$-th spins [3]. In particular, for spin $1 / 2(m=2)$ we have

$$
S_{i j}=\frac{1}{2}\left(1+\boldsymbol{\sigma}_{i} \cdot \boldsymbol{\sigma}_{j}\right),
$$

where $\boldsymbol{\sigma}_{k} \equiv\left(\sigma_{k}^{x}, \sigma_{k}^{y}, \sigma_{k}^{z}\right)$ denotes the three Pauli matrices acting on the $k$-th spin. In fact, there is also a natural $\mathrm{su}(m \mid n)$ supersymmetric version of the HS chain, which has been extensively studied in the literature [4-6].

The HS chain possesses remarkable physical and mathematical properties. Indeed, it is intimately related to the one-dimensional Hubbard model with long-range hopping, from which it is obtained in the limit of infinite on-site interaction at half filling [7]. It has also been proposed as the simplest model providing an explicit realization of fractional statistics and anyons in one dimension [8-11]. As to its mathematical properties, the HS chain is completely integrable [12], can be solved via an asymptotic Bethe ansatz [13-15], is one of the few models invariant under the Yangian group for a finite number of sites [11], and its partition function can be evaluated in closed form [16] via Polychronakos's "freezing trick" [17].

In fact, the HS chain (1.1) is essentially a limiting case of a more general model due to Inozemtsev [18], whose Hamiltonian we shall take as

$$
H=J \sum_{i<j} \wp_{N}(i-j)\left(1-S_{i j}\right) .
$$

Here

$$
\wp_{N}(x) \equiv \wp\left(x ; \frac{N}{2}, \frac{\mathrm{i} \alpha}{2}\right)
$$

denotes the Weierstrass elliptic function [19] with periods $N$ and i $\alpha$, with $\alpha>0$. Indeed, since

$$
\lim _{\alpha \rightarrow \infty} \wp_{N}(x)=\frac{\pi^{2}}{N^{2}}\left(\sin ^{-2}\left(\frac{\pi x}{N}\right)-\frac{1}{3}\right)
$$

(see Appendix A), the $\alpha \rightarrow \infty$ limit of the Hamiltonian (1.2) differs from (1.1) by a constant multiple of the operator $\sum_{i<j}\left(1-S_{i j}\right)$, which trivially commutes with the HS 
chain Hamiltonian. More surprising is the fact that when $\alpha \rightarrow 0$ the Inozemtsev chain is related to the Heisenberg chain

$$
H=J \sum_{i=1}^{N}\left(1-S_{i, i+1}\right), \quad S_{N, N+1} \equiv S_{1 N}
$$

(cf. [18]). More precisely, if $1 \leqslant x \leqslant N-1$ we have

$$
\lim _{\alpha \rightarrow 0} \mathrm{e}^{2 \pi / \alpha}\left(\frac{\alpha^{2}}{4 \pi^{2}} \wp_{N}(x)-\frac{1}{12}\right)=\delta_{1, x}+\delta_{N-1, x}
$$

(see again Appendix A).

Interestingly, in the last decade the Inozemtsev chain has also received considerable attention in the context of the AdS/CFT correspondence [20-23]. This unexpected connection stems from the work of Minahan and Zarembo [24], who showed that at one loop the (planar) spectrum of the dilation operator of $\mathcal{N}=4$ super Yang-Mills gauge theory can be generated by a suitable integrable spin chain with nearest-neighbor interactions. In order to generalize the latter result to more than one loop, it becomes necessary to consider integrable spin chains with long-range interactions. Moreover, any such chain describing $\mathcal{N}=4$ Berenstein-Maldacena-Nastase theory non-perturbatively should contain an additional parameter related to the Yang-Mills coupling constant. As first noted by Serban and Staudacher [25], the Inozemtsev chain (1.2) is one of the simplest models fulfilling the last two requirements. Since this chain is also generally believed to be integrable, it has been extensively studied as a candidate for generating the planar spectrum of the dilation operator at several loops [26]. It should be noted, however, that a complete rigorous proof of the integrability of the Inozemtsev chain has not yet been found, in spite of several promising partial results in this direction $[18,27]$. In any case, the energy spectrum of this chain is not explicitly known beyond the twomagnon sector.

In this paper we introduce an $\mathrm{su}(1 \mid 1)$ elliptic chain, which is in fact the simplest supersymmetric version of the Inozemtsev model (1.2). We show that, rather unexpectedly, this chain is completely integrable and its whole spectrum can be computed in closed form. Our proof is based on two key ideas. In the first place, we exploit the well-known fact that the $\mathrm{su}(1 \mid 1)$ permutation operators can be expressed in terms of annihilation and creation operators of a single species of spinless fermions. This implies that the $\mathrm{su}(1 \mid 1)$ elliptic chain is equivalent to a model of hopping free fermions, which smoothly interpolates between the su(1|1) HS chain and the standard $X X$ model (at a critical value of the magnetic field). The second ingredient in our proof is the explicit computation of the dispersion relation of the $\mathrm{su}(1 \mid 1)$ elliptic chain using standard techniques in analytic function theory $[18,27,28]$.

From the explicit knowledge of the dispersion relation, we have been able to study several properties of the $\mathrm{su}(1 \mid 1)$ elliptic chain in the thermodynamic limit. In the first place, we have analyzed the low momentum behavior of the dispersion relation, showing that the energy is quadratic in the momentum near $p=0$ for all finite values of the chain's parameter $\alpha$. Thus, at low energies the $\mathrm{su}(1 \mid 1)$ elliptic chain behaves as a 
critical $X X$ model, and in particular its low energy excitations cannot be described by an effective two-dimensional conformal field theory. By contrast, it is well known that the dispersion relation of the $\mathrm{su}(1 \mid 1) \mathrm{HS}$ chain is linear near the origin, and at low energies its spectrum coincides with that of a conformal field theory of $m$ noninteracting Dirac fermions with only positive energies [29]. We have next computed the chain's thermodynamic functions in closed form, and determined their low temperature limit. Our analysis shows that at low temperatures the su(1|1) elliptic chain behaves essentially as a critical $X X$ model, and markedly differs from both the $\mathrm{su}(1 \mid 1)$ and $\mathrm{su}(2)$ HS chains. We have also studied the asymptotic behavior of the level density as the number of sites tends to infinity, proving that it approaches a Gaussian distribution with parameters equal to the mean and the standard deviation of the spectrum, as is typically the case for spin chains of HS type [6,30-32]. Finally, we have introduced the $\mathrm{su}(1 \mid 1)$ supersymmetric analog of Inozemtsev's infinite (hyperbolic) chain, showing that its dispersion relation is proportional to the thermodynamic limit of the $\mathrm{su}(1 \mid 1)$ elliptic chain's dispersion relation.

The paper is organized as follows. In Section 2 we introduce the su(1|1) elliptic chain and, as explained above, solve it by transforming it into a system of hopping fermions whose dispersion relation we compute in closed form. Section 3 is devoted to the derivation of the chain's thermodynamic functions and the analysis of the asymptotic behavior of its level density. Section 4 deals with the su(1|1) version of Inozemtsev's infinite hyperbolic chain and its complete solution. In Section 5 we summarize the paper's main results, and point out several future developments suggested by our work. The paper ends with two technical appendices on the computation of several limits involving elliptic Weierstrass functions, and on the proof of the properties of quasiperiodic functions needed for the evaluation of the dispersion relation of the $\operatorname{su}(1 \mid 1)$ elliptic chain.

\section{The $\mathrm{su}(1 \mid 1)$ elliptic chain and its solution}

The model we shall study is the $\mathrm{su}(1 \mid 1)$ version of Inozemtsev's chain (1.2), in which each site is occupied by a spinless particle which can be either a boson or a fermion. Thus the chain's Hamiltonian can be written as

$$
H=J \sum_{i<j} h(i-j)\left(1-\mathscr{S}_{i j}\right),
$$

where, as before,

$$
h(x)=\wp_{N}(x),
$$

and the $\mathscr{S}_{i j}$ are the standard su(1|1) permutation operators. More precisely, if we denote by $|0\rangle$ and $|1\rangle$ respectively the boson and the fermion states, the standard basis of the chain's Hilbert space consists of the $2^{N}$ product states

$$
\left|s_{1}\right\rangle \otimes \cdots \otimes\left|s_{N}\right\rangle \equiv\left|s_{1}, \ldots, s_{N}\right\rangle, \quad s_{i} \in\{0,1\} .
$$


We then have

$$
\mathscr{S}_{i j}\left|s_{1}, \ldots, s_{i}, \ldots, s_{j}, \ldots, s_{N}\right\rangle=(-1)^{n}\left|s_{1}, \ldots, s_{j}, \ldots, s_{i}, \ldots, s_{N}\right\rangle,
$$

where $n=s_{i}=s_{j}$ if $s_{i}=s_{j}$, while $n$ is equal to the number of fermions occupying the sites $i+1, \ldots, j-1$ if $s_{i} \neq s_{j}$. Equivalently,

$$
\mathscr{S}_{i j}=b_{i}^{\dagger} b_{j}^{\dagger} b_{i} b_{j}+f_{i}^{\dagger} f_{j}^{\dagger} f_{i} f_{j}+b_{i}^{\dagger} f_{j}^{\dagger} f_{i} b_{j}+f_{i}^{\dagger} b_{j}^{\dagger} b_{i} f_{j},
$$

where $b_{k}^{\dagger}$ and $f_{k}^{\dagger}$ respectively denote the boson and fermion creation operators acting on the $k$-th site. It is important to note that, since each site is occupied by either one boson or one fermion, the chain's Hilbert space is the subspace $\mathcal{H}$ of the Fock space determined by the constraints

$$
b_{i}^{\dagger} b_{i}+f_{i}^{\dagger} f_{i}=1, \quad i=1, \ldots, N .
$$

It can be shown that a chain of the form $(2.1 a)$ with arbitrary $h$ can be mapped to a system of $N$ "hopping" (spinless) free fermions. The key idea is to regard the bosonic state $|0\rangle$ as the vacuum for the fermion. More formally, we define the operators

$$
a_{i}^{\dagger} \equiv f_{i}^{\dagger} b_{i}, \quad i=1, \ldots, N,
$$

which satisfy the canonical anticommutation relations on $\mathcal{H}$ on account of the constraints (2.2). Thus $a_{i}^{\dagger}$ creates a fermion at the site $i$. The chain's sites can now be either empty or occupied by a fermion, and the Hilbert space $\mathcal{H}$ is identified with the whole Fock space for the new system of fermions. As first noted by Haldane [4], the $\mathrm{su}(1 \mid 1)$ permutation operator $\mathscr{S}_{i j}$ can be expressed in terms of the fermionic operators $a_{k}, a_{k}^{\dagger}$ as

$$
\mathscr{S}_{i j}=1-a_{i}^{\dagger} a_{i}-a_{j}^{\dagger} a_{j}+a_{i}^{\dagger} a_{j}+a_{j}^{\dagger} a_{i}
$$

(see Ref. [33] for more details). Substituting (2.3) into (2.1a) we easily obtain

$$
H=J \sum_{i \neq j} h(|i-j|) a_{i}^{\dagger}\left(a_{i}-a_{j}\right)
$$

which is indeed the Hamiltonian of a system of $N$ free hopping fermions.

The Weierstrass function $h(x)=\wp_{N}(x)$ is even and $N$-periodic, i.e,

$$
h(x)=h(-x)=h(x+N), \quad \forall x .
$$

Hence

$$
h(x)=h(N-x), \quad \forall x,
$$

so that the model (2.1) is translation-invariant. Equivalently, the chain sites can be viewed as $N$ equidistant points lying on a circle, as in the case of the HaldaneShastry chain. When the chain $(2.1 a)$ is translation-invariant, i.e., when the function $h$ satisfies (2.6), the diagonal terms in Eq. (2.4) can be considerably simplified. Indeed, in this case the coefficient of $a_{i}^{\dagger} a_{i}$ is given by

$$
\sum_{j ; j \neq i} h(|i-j|)=\sum_{l=1}^{i-1} h(l)+\sum_{l=1}^{N-i} h(l)=\sum_{l=1}^{i-1} h(l)+\sum_{l=i}^{N-1} h(N-l)=\sum_{l=1}^{N-1} h(l),
$$


independently of $i$, so that we can write

$$
H=-J \sum_{i, j=1}^{N} h(|i-j|) a_{i}^{\dagger} a_{j}
$$

provided that we set

$$
h(0) \equiv-\sum_{l=1}^{N-1} h(l) .
$$

Thus a translation-invariant chain (2.1a)-(2.6) is equivalent to a system of $N$ free hopping fermions lying on a circle, with hopping amplitude between the $i$-th and $j$ th sites equal to $h(|i-j|)$.

When $h$ satisfies Eq. (2.6), the Hamiltonian (2.8) can be diagonalized by performing the discrete Fourier transform

$$
c_{l}=\frac{1}{\sqrt{N}} \sum_{k=1}^{N} \mathrm{e}^{-2 \pi \mathrm{i} k l / N} a_{k}, \quad l=0,1, \ldots, N-1 .
$$

Indeed, first of all it is immediate to check that the operators $c_{l}$ satisfy the canonical anticommutation relations, on account of the unitarity of the mapping (2.10); in fact, we shall show below that $c_{l}^{\dagger}$ creates a fermion with momentum $2 \pi l / N(\bmod 2 \pi)$. Using the inverse Fourier transform formula

$$
a_{k}=\frac{1}{\sqrt{N}} \sum_{l=0}^{N-1} \mathrm{e}^{2 \pi \mathrm{i} k l / N} c_{l}, \quad k=1, \ldots, N,
$$

in the Hamiltonian (2.8) we obtain

$$
H=J \sum_{l, m=0}^{N-1} h_{l m} c_{l}^{\dagger} c_{m}
$$

with

$$
h_{l m}=-\frac{1}{N} \sum_{j, k=1}^{N} h(|k-j|) \mathrm{e}^{2 \pi \mathrm{i}(j m-k l) / N} .
$$

Using the translation invariance condition (2.6), it is straightforward to show that

$$
h_{l m}=-\frac{1}{N} \sum_{s=0}^{N-1} \mathrm{e}^{-2 \pi \mathrm{i} s l / N} h(s) \sum_{j=1}^{N} \mathrm{e}^{2 \pi \mathrm{i} j(m-l) / N}=\delta_{l m} \varepsilon_{l},
$$

where

$$
\varepsilon_{l}=-\sum_{s=0}^{N-1} \mathrm{e}^{-2 \pi \mathrm{i} s l / N} h(s)
$$

Thus

$$
H=J \sum_{l=0}^{N-1} \varepsilon_{l} c_{l}^{\dagger} c_{l}
$$


is indeed diagonal when written in terms of the Fourier-transformed operators $c_{l}$. The dispersion relation $\varepsilon_{l}$ can be further simplified using the definition of $h(0)$ and Eq. (2.6), namely

$$
\begin{aligned}
\varepsilon_{l} & =-h(0)-\sum_{j=1}^{N-1} \mathrm{e}^{-2 \pi \mathrm{i} j l / N} h(j)=\sum_{j=1}^{N-1}\left(1-\mathrm{e}^{-2 \pi \mathrm{i} j l / N}\right) h(j) \\
& =\sum_{j=1}^{N-1}\left(1-\mathrm{e}^{2 \pi \mathrm{i} j l / N}\right) h(j)=\sum_{j=1}^{N-1}(1-\cos (2 \pi j l / N)) h(j) .
\end{aligned}
$$

Remark 1. From the latter equation it follows that

$$
\varepsilon_{0}=0, \quad \varepsilon_{l}=\varepsilon_{N-l} .
$$

By the first of these identities and Eq. (2.11), the degeneracy of each energy level is even, as required by the $\mathrm{su}(1 \mid 1)$ symmetry.

Remark 2. Another immediate consequence of Eq. (2.11) is the complete integrability of the model (2.1a)-(2.6), since the number operators $c_{l}^{\dagger} c_{l}(l=0, \ldots, N-1)$ are a commuting family of first integrals.

Remark 3. Consider the translation operator $T$, defined on the basis states by

$$
T\left|s_{1}, \ldots, s_{N}\right\rangle=\left|s_{2}, \ldots, s_{N}, s_{1}\right\rangle \text {. }
$$

It is easy to check that $T$ is characterized by the relations

$$
T^{-1} a_{j} T=a_{j+1}, \quad j=1, \ldots, N ; \quad T|0\rangle=|0\rangle,
$$

where $|0\rangle \equiv|0, \ldots, 0\rangle$ denotes the vacuum state. The momentum operator $P$ is defined (up to integer multiples of $2 \pi$ ) in the usual way:

$$
T=\mathrm{e}^{\mathrm{i} P} .
$$

It is straightforward to check that when the function $h$ satisfies Eq. (2.6) the Hamiltonian (2.8) commutes with $T$, and hence with $P$. It turns out that $P$ is also diagonalized by the Fourier transform. Indeed, the conditions (2.13) can be rewritten in terms of the Fourier transformed operators $c_{l}$ as

$$
T^{-1} c_{l} T=\mathrm{e}^{2 \pi \mathrm{i} l / N} c_{l}, \quad l=0, \ldots, N-1 .
$$

Substituting the ansatz

$$
P=\sum_{l=0}^{N-1} p_{l} c_{l}^{\dagger} c_{l}
$$

into the previous equations we readily obtain

$$
p_{l}=\frac{2 \pi l}{N} \quad(\bmod 2 \pi),
$$

so that the momentum operator $P$ is given by

$$
P=\frac{2 \pi}{N} \sum_{l=0}^{N-1} l c_{l}^{\dagger} c_{l} \quad(\bmod 2 \pi)
$$

(cf. Ref. [29]). Thus the state created by $c_{l}^{\dagger}$ has well-defined energy $\varepsilon_{l}$ and momentum $2 \pi l / N(\bmod 2 \pi)$, as we had anticipated. 
As we have just seen, in order to solve the elliptic chain (2.1) we need to evaluate in closed form the sum in Eq. (2.12) when $h(x)=\wp_{N}(x)$. In fact, the sum $\sum_{j=1}^{N} \wp_{N}(j)$ was computed in Ref. [28], with the result

$$
\sum_{j=1}^{N-1} \wp_{N}(j)=\frac{2}{\mathrm{i} \alpha}\left[\eta_{3}\left(\frac{1}{2}, \frac{\mathrm{i} \alpha}{2}\right)-N \eta_{3}\left(\frac{N}{2}, \frac{\mathrm{i} \alpha}{2}\right)\right] .
$$

Here we have used the standard notation

$$
\eta_{i}\left(\omega_{1}, \omega_{3}\right) \equiv \zeta\left(\omega_{i} ; \omega_{1}, \omega_{3}\right)
$$

where $\zeta\left(z ; \omega_{1}, \omega_{3}\right)$ denotes the Weierstrass zeta function associated to the lattice $2 m \omega_{1}+2 n \omega_{3}$, with $m, n \in \mathbb{Z}$ and $\operatorname{Im}\left(\omega_{3} / \omega_{1}\right)>0$ (cf. [19]). It thus suffices to compute the discrete Fourier transform of the Weierstrass function $\wp_{N}(x)$. This can be done using a technique due to Inozemtsev [18,27], which we shall briefly summarize for the reader's convenience.

For fixed $l=1,2, \ldots, N-1$, we define the function

$$
f_{l}(z)=\sum_{j=0}^{N-1} \mathrm{e}^{-2 \pi \mathrm{i} j l / N} \wp_{N}(z+j) .
$$

From the periodicity of the Weierstrass elliptic function, it is easy to check that $f_{l}$ satisfies the quasi-periodicity conditions in Eq. (B.1) with

$$
2 \omega_{1}=1, \quad 2 \omega_{3}=\mathrm{i} \alpha, \quad p=l / N .
$$

On the other hand, $f_{l}$ is clearly analytic everywhere except at points congruent to the origin, i.e., on the lattice $m+\mathrm{i} n \alpha(m, n \in \mathbb{Z})$. In fact, the only term in the sum defining $f_{l}$ which is singular at the origin is the one with $j=0$. Since $\dagger$

$$
\wp\left(z ; \omega_{1}, \omega_{3}\right)=\frac{1}{z^{2}}+\mathrm{O}\left(z^{2}\right)
$$

the Laurent series of $f_{l}$ about $z=0$ is simply

$$
f_{l}(z)=\frac{1}{z^{2}}+\mathrm{O}(1)
$$

and we can therefore apply Eqs. (B.2) and (B.5) with $\omega_{3}=\mathrm{i} \alpha / 2$ and $p=l / N$. We thus have

$$
\lim _{z \rightarrow 0}\left(f_{l}(z)-\frac{1}{z^{2}}\right)=\frac{1}{2} \wp_{1}\left(\frac{\mathrm{i} \alpha l}{N}\right)-\frac{1}{2}\left(\zeta_{1}\left(\frac{\mathrm{i} \alpha l}{N}\right)-\frac{2 l}{N} \zeta_{1}\left(\frac{\mathrm{i} \alpha}{2}\right)\right)^{2},
$$

where $\wp_{1}$ and $\zeta_{1}$ are the Weierstrass functions with half-periods $1 / 2$ and $i \alpha / 2$. On the other hand, from the definition of $f_{l}$ and Eq. (2.15) we easily obtain

$$
f_{l}(z)=\frac{1}{z^{2}}+\sum_{j=1}^{N-1} \mathrm{e}^{-2 \pi \mathrm{i} j l / N} \wp_{N}(j)+\mathrm{O}(z) .
$$

$\dagger$ We shall write $f(z)=\mathrm{O}(g(z))$ for $z \rightarrow z_{0}$ if there is a positive constant $C$ such that $|f(z)| \leqslant C|g(z)|$ for $z$ sufficiently close to $z_{0}$. 
Comparing with Eq. (2.16) we conclude that

$$
\sum_{j=1}^{N-1} \mathrm{e}^{-2 \pi \mathrm{i} j l / N} \wp_{N}(j)=\frac{1}{2} \wp_{1}\left(\frac{\mathrm{i} \alpha l}{N}\right)-\frac{1}{2}\left(\zeta_{1}\left(\frac{\mathrm{i} \alpha l}{N}\right)-\frac{2 l}{N} \zeta_{1}\left(\frac{\mathrm{i} \alpha}{2}\right)\right)^{2} .
$$

Combining this formula with Eq. (2.14) we obtain the following explicit expression for the dispersion relation $\varepsilon_{l}$ for $l=1, \ldots, N-1$ :

$\varepsilon_{l}=\frac{2}{\mathrm{i} \alpha}\left[\zeta_{1}\left(\frac{\mathrm{i} \alpha}{2}\right)-N \eta_{3}\left(\frac{N}{2}, \frac{\mathrm{i} \alpha}{2}\right)\right]-\frac{1}{2}\left[\wp_{1}\left(\frac{\mathrm{i} \alpha l}{N}\right)-\left(\zeta_{1}\left(\frac{\mathrm{i} \alpha l}{N}\right)-\frac{2 l}{N} \zeta_{1}\left(\frac{\mathrm{i} \alpha}{2}\right)\right)^{2}\right]$

(and, of course, $\varepsilon_{0}=0$ ). This formula can be further simplified with the help of the homogeneity properties of the Weierstrass functions, namely

$\wp\left(\lambda z ; \lambda \omega_{1}, \lambda \omega_{3}\right)=\frac{1}{\lambda^{2}} \wp\left(z ; \omega_{1}, \omega_{3}\right), \quad \zeta\left(\lambda z ; \lambda \omega_{1}, \lambda \omega_{3}\right)=\frac{1}{\lambda} \zeta\left(z ; \omega_{1}, \omega_{3}\right)$.

Using these formulas with $\lambda=\mathrm{i} \alpha$ we easily arrive at the following closed-form expression for the dispersion relation of the $\mathrm{su}(1 \mid 1)$ elliptic chain (2.1):

$\varepsilon_{l}=\frac{1}{2 \alpha^{2}}\left[\wp(l / N)-\left(\zeta(l / N)-2 \eta_{1} \frac{l}{N}\right)^{2}+4\left(N \hat{\eta}_{1}-\eta_{1}\right)\right], \quad l=1, \ldots, N-1$,

where from now on

$$
\wp(z) \equiv \wp\left(z ; \frac{1}{2}, \frac{\mathrm{i}}{2 \alpha}\right), \quad \zeta(z) \equiv \zeta\left(z ; \frac{1}{2}, \frac{\mathrm{i}}{2 \alpha}\right)
$$

shall denote the Weierstrass functions with periods 1 and $\mathrm{i} / \alpha$, and we have also set

$$
\eta_{1} \equiv \eta_{1}\left(\frac{1}{2}, \frac{\mathrm{i}}{2 \alpha}\right), \quad \hat{\eta}_{1} \equiv \eta_{1}\left(\frac{1}{2}, \frac{\mathrm{i} N}{2 \alpha}\right) .
$$

Remark 4. The reader may have noticed that Eq. (2.17) coincides with the dispersion relation of the 1 -magnons with momentum $2 \pi l / N(\bmod 2 \pi)$ of the $\operatorname{su}(2)$ elliptic chain (1.2) computed in Ref. [18]. In fact, this remarkable relation between the $\mathrm{su}(2)$ and $\mathrm{su}(1 \mid 1)$ elliptic chains holds for an arbitrary translation-invariant chain of the form $(2.1 a)$. This is a further indication of the greater simplicity of the su(1|1) models compared to their $\mathrm{su}(2)$ counterparts.

All the terms in the dispersion relation (2.19) depend on $N$ through the combination $l / N$ except for the one involving $\hat{\eta}_{1}$, which can be eliminated through the shift $h(x) \mapsto h(x)-\left(2 \hat{\eta}_{1} / \alpha^{2}\right)$. In fact, it is possible to combine this shift with an appropriate rescaling so that the $\alpha \rightarrow \infty$ and $\alpha \rightarrow 0$ limits of the chain $(2.1 a)$ exactly coincide with the $\mathrm{su}(1 \mid 1)$ versions of the Haldane-Shastry and Heisenberg chains, i.e., Eqs. (1.1) and (1.4) with $S_{i j}$ replaced by $\mathscr{S}_{i j}$. Indeed, it suffices to take [34]

$$
h(x)=\left(\frac{\alpha}{\pi}\right)^{2} \sinh ^{2}\left(\frac{\pi}{\alpha}\right)\left(\wp_{N}(x)-\frac{2 \hat{\eta}_{1}}{\alpha^{2}}\right) ;
$$

see Appendix A for the details. For this reason, we shall from now on adopt the normalization (2.20) when referring to the su(1|1) elliptic chain (2.1a). By Eq. (2.12), the corresponding dispersion relation is simply $\ddagger$

$$
\varepsilon_{l}=\mathcal{E}(l / N),
$$

$\ddagger$ It can be easily checked (cf. Eq. (2.25) below) that $\mathcal{E}(0) \equiv \lim _{p \rightarrow 0} \mathcal{E}(p)=0$. 
where

$$
\mathcal{E}(p)=\frac{\sinh ^{2}(\pi / \alpha)}{2 \pi^{2}}\left[\wp(p)-\left(\zeta(p)-2 \eta_{1} p\right)^{2}-4 \eta_{1}\right]
$$

is independent of $N$, and $p$ is the physical moment in units of $2 \pi$.

Remark 5. It should be noted that the rescaled interaction strength $h(x)$ in Eq. (2.20) remains positive for $0<x<N$, so that the chain (2.1a)-(2.20) is of ferromagnetic type for $J>0$ (cf. Fig. 1). Indeed, the function $\wp_{N}(x)$ has an absolute minimum in the interval $0<x<N$ at the real half-period $x=N / 2$, and thus it suffices to show that

$$
\wp_{N}(N / 2)-\frac{2 \hat{\eta}_{1}}{\alpha^{2}}>0 \text {. }
$$

From Eq. (A.1) with $\omega_{1}=\mathrm{i} \alpha / 2, \omega_{3}=-N / 2$ (so that $\operatorname{Im}\left(\omega_{3} / \omega_{1}\right)=N / \alpha>0$ ) and the homogeneity of the Weierstrass zeta function (cf. the second Eq. (2.18)) we easily obtain

$$
\begin{aligned}
\wp_{N}(N / 2)-\frac{2 \hat{\eta}_{1}}{\alpha^{2}} & =\wp_{N}(N / 2)+\frac{2}{\mathrm{i} \alpha} \eta_{1}(\mathrm{i} \alpha / 2,-N / 2) \equiv \wp\left(\omega_{3} ; \omega_{1}, \omega_{3}\right)+\frac{1}{\omega_{1}} \eta_{1}\left(\omega_{1}, \omega_{3}\right) \\
& =\frac{\pi^{2}}{\alpha^{2}} \sinh ^{-2}\left(\frac{N \pi}{2 \alpha}\right)+\frac{4 \pi^{2}}{\alpha^{2}} \sum_{n=1}^{\infty} n \mathrm{e}^{-n N \pi / \alpha} \operatorname{coth}\left(\frac{n N \pi}{\alpha}\right)>0 .
\end{aligned}
$$

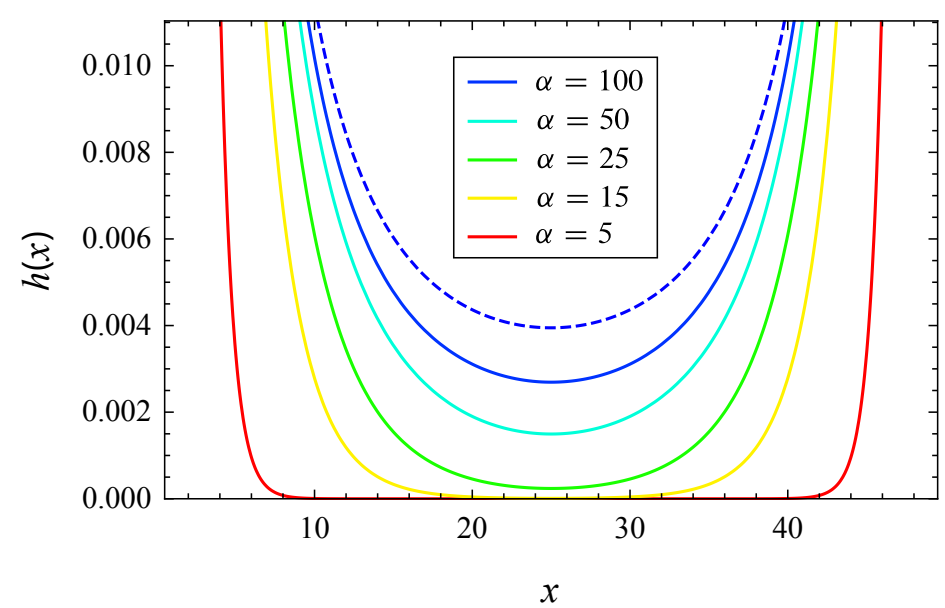

Figure 1. (Color online) Interaction strength $h(x)$ in Eq. (2.20) for $N=50$ spins and several values of $\alpha$ in the range $[5,100]$. The dashed blue line represents the interaction strength $h(x)=(\pi / 50)^{2} \sin ^{-2}(\pi x / 50)$ of the Haldane-Shastry chain with 50 spins $(\alpha=\infty)$.

It should be expected (and can, in fact, be analytically proved; see Appendix A for the details) that the $\alpha \rightarrow \infty$ and $\alpha \rightarrow 0$ limits of Eq. (2.21b) respectively yield the dispersion relations of the $\mathrm{su}(1 \mid 1)$ Haldane-Shastry and Heisenberg chains. In fact, the dispersion relation of the $\mathrm{su}(1 \mid 1)$ HS chain was computed in Ref. [5] essentially by the same procedure followed here, with the result

$$
\mathcal{E}(p)=2 \pi^{2} p(1-p) .
$$




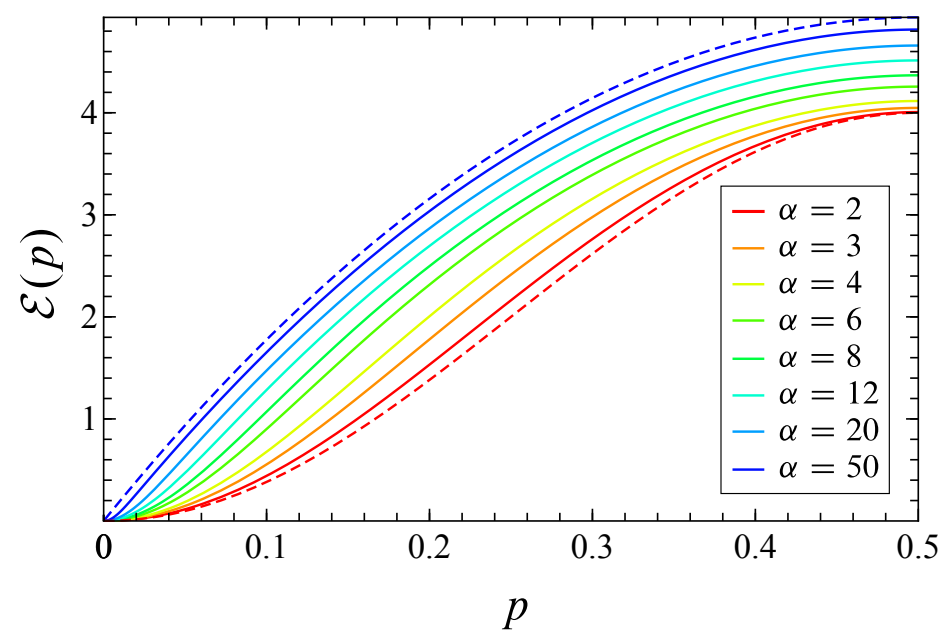

Figure 2. (Color online). Solid lines: dispersion relation (2.21b) of the elliptic su(1|1) chain (2.1a)-(2.20) for several values of the parameter $\alpha$ between 2 (bottom) and 50 (top). Dashed lines: dispersion relations of the the critical $X X$ model (2.23) (bottom) and the $\mathrm{su}(1 \mid 1)$ Haldane-Shastry chain (top). Only the range $0 \leqslant p \leqslant 1 / 2$ has been shown, since $\mathcal{E}(p)=\mathcal{E}(1-p)$ on account of $(2.12)$.

As to the $\mathrm{su}(1 \mid 1)$ Heisenberg chain, note first of all that from Eq. (2.8) with $h(x)=$ $\delta_{1, x}+\delta_{N-1, x}$ (and thus $h(0)=-2$, by Eq. (2.9)) it immediately follows that the Hamiltonian of this model can be expressed as

$$
H=J\left[2 \sum_{i=1}^{N} a_{i}^{\dagger} a_{i}-\sum_{i=1}^{N}\left(a_{i}^{\dagger} a_{i+1}+a_{i+1}^{\dagger} a_{i}\right)\right], \quad a_{N+1} \equiv a_{1} .
$$

It is well known [35] that the latter Hamiltonian is transformed by the standard JordanWigner transformation

$$
a_{k}=\sigma_{1}^{z} \cdots \sigma_{k-1}^{z} \cdot \frac{1}{2}\left(\sigma_{k}^{x}-\mathrm{i} \sigma_{k}^{y}\right), \quad k=1, \ldots, N,
$$

into the $X X$ model (at a critical value of the magnetic field)

$$
H=J\left[\frac{1}{2} \sum_{i=1}^{N}\left(\sigma_{i}^{x} \sigma_{i+1}^{x}+\sigma_{i}^{y} \sigma_{i+1}^{y}\right)+\sum_{i=1}^{N}\left(1+\sigma_{i}^{z}\right)\right], \quad \boldsymbol{\sigma}_{N+1} \equiv \boldsymbol{\sigma}_{1} .
$$

It follows from the previous discussion that the $\alpha \rightarrow 0$ limit of the su(1|1) elliptic chain $(2.1 a)-(2.20)$ is equivalent to a critical $X X$ model, a fact that is not obvious at all a priori. From Eq. (2.12) with $h(x)=\delta_{1, x}+\delta_{N-1, x}$, it is straightforward to obtain the well-known dispersion relation of this model

$$
\mathcal{E}(p)=4 \sin ^{2}(\pi p) .
$$

As expected, the dispersion relation $(2.21 b)$ varies smoothly between its limits $(2.24)$ and (2.22) as $\alpha$ ranges from 0 to $\infty$; see, e.g., Fig. 2.

Let us next briefly analyze the low momentum behavior of the dispersion relation $(2.21 b)$. To this end, we recall the Laurent series $\wp(z)=\frac{1}{z^{2}}+\frac{g_{2}}{20} z^{2}+\frac{g_{3}}{28} z^{4}+\mathrm{O}\left(z^{6}\right), \quad \zeta(z)=\frac{1}{z}-\frac{g_{2}}{60} z^{3}-\frac{g_{3}}{140} z^{5}+\mathrm{O}\left(z^{7}\right)$, 
where $g_{i} \equiv g_{i}(1 / 2, \mathrm{i} /(2 \alpha))$ are the invariants of the Weierstrass function with periods $(1, \mathrm{i} / \alpha)$ (see, e.g., [19]). Expanding Eq. (2.21b) around $p=0$ with the help of the previous formulas we readily obtain

$$
\mathcal{E}(p)=\frac{p^{2}}{2 m(\alpha)}+O\left(p^{4}\right)
$$

where the effective mass $m(\alpha)$ is given by

$$
m(\alpha)=\frac{12 \pi^{2}}{\left(g_{2}-48 \eta_{1}^{2}\right) \sinh ^{2}(\pi / \alpha)} .
$$

As $\mathcal{E}(p)=\mathcal{E}(1-p)$, a similar formula is valid around $p=1$, with $p$ replaced by $1-p$. In particular, since the low-energy dispersion relation is not linear in the momentum, the low energy excitations cannot be described by an effective two-dimensional conformal field theory. By contrast, it is well known that the low energy excitations of the $\mathrm{su}(m \mid 1)$ supersymmetric Haldane-Shastry spin chain coincide with the spectrum of a conformal field theory of $m$ non-interacting Dirac fermions with only positive energies [29].

\section{Thermodynamics}

\subsection{Thermodynamic functions}

Using the dispersion relation $(2.21 b)$, it is straightforward to evaluate the free energy per site

$$
f(T)=-\frac{1}{\beta} \lim _{N \rightarrow \infty} \frac{\log \mathcal{Z}_{N}}{N}, \quad \beta \equiv \frac{1}{k_{\mathrm{B}} T},
$$

where $\mathcal{Z}_{N}$ is the partition function for $N$ spins. Indeed, since the model in momentum space is equivalent to a system of $N$ free fermions with energies $\varepsilon_{l}=J \mathcal{E}(l / N)$, the partition function is given by

$$
\mathcal{Z}_{N}=\prod_{l=0}^{N-1}\left(1+\mathrm{e}^{-J \beta \mathcal{E}(l / N)}\right)
$$

and therefore

$$
f(T)=-\frac{1}{\beta} \lim _{N \rightarrow \infty} \sum_{l=0}^{N-1} \frac{1}{N} \log \left(1+\mathrm{e}^{-J \beta \mathcal{E}(l / N)}\right)=-\frac{1}{\beta} \int_{0}^{1} \log \left(1+\mathrm{e}^{-J \beta \mathcal{E}(p)}\right) \mathrm{d} p .
$$

The remaining thermodynamic functions, i.e., the energy, the specific heat, and the entropy (per site), respectively given by

$$
u=\frac{\partial}{\partial \beta}(\beta f), \quad c=\frac{\partial u}{\partial T}, \quad s=\frac{1}{T}(u-f),
$$

can be readily computed in closed form using the previous expression for $f(T)$. More precisely, introducing the dimensionless temperature $\tau \equiv 1 /(|J| \beta)$ we obtain:

$$
\frac{1}{|J|}\left(f-u_{0}\right)=-\tau \int_{0}^{1} \log \left(1+\mathrm{e}^{-\mathcal{E}(p) / \tau}\right) \mathrm{d} p
$$




$$
\begin{aligned}
& \frac{1}{|J|}\left(u-u_{0}\right)=\int_{0}^{1} \frac{\mathcal{E}(p)}{1+\mathrm{e}^{\mathcal{E}(p) / \tau}} \mathrm{d} p \\
& \frac{c}{k_{\mathrm{B}}}=\frac{1}{4 \tau^{2}} \int_{0}^{1} \mathcal{E}^{2}(p) \operatorname{sech}^{2}\left(\frac{\mathcal{E}(p)}{2 \tau}\right) \mathrm{d} p \\
& \frac{s}{k_{\mathrm{B}}}=\int_{0}^{1} \varphi\left(\frac{\mathcal{E}(p)}{2 \tau}\right) \mathrm{d} p
\end{aligned}
$$

where $\varphi(x) \equiv \log (2 \cosh x)-x \tanh x$ and

$$
u_{0} \equiv u(0)=f(0)=\frac{1}{2}(J-|J|) \int_{0}^{1} \mathcal{E}(p) \mathrm{d} p .
$$

Remarkably, the last integral can be evaluated in closed form, with the result

$$
\int_{0}^{1} \mathcal{E}(p)=\frac{2}{\pi^{2}} \sinh ^{2}(\pi / \alpha)\left(\frac{\pi^{2}}{6}-\eta_{1}\right)
$$

see the next subsection for more details. Likewise, the density of fermions is given by

$$
\nu_{\mathrm{F}}=\lim _{N \rightarrow \infty} \frac{1}{N} \sum_{l=0}^{N-1}\left(1+\mathrm{e}^{J \beta \mathcal{E}(l / N)}\right)^{-1}=\int_{0}^{1} \frac{\mathrm{d} p}{1+\mathrm{e}^{J \beta \mathcal{E}(p)}},
$$

or, in terms of the dimensionless temperature $\tau$,

$$
\frac{J}{|J|}\left(\nu_{\mathrm{F}}-\frac{1}{2}\right)+\frac{1}{2}=\int_{0}^{1} \frac{\mathrm{d} p}{1+\mathrm{e}^{\mathcal{E}(p) / \tau}} .
$$

The low temperature behavior of the thermodynamic functions can be readily deduced from the previous formulas. Indeed, performing the change of variables $x=\mathcal{E}(p) / \tau$ in Eq. (3.2) we obtain

$$
\frac{1}{|J|}\left(f-u_{0}\right)=-2 \tau \int_{0}^{1 / 2} \log \left(1+\mathrm{e}^{-\mathcal{E}(p) / \tau}\right) \mathrm{d} p=-2 \tau^{2} \int_{0}^{\mathcal{E}(1 / 2) / \tau} \log \left(1+\mathrm{e}^{-x}\right) \frac{\mathrm{d} x}{\mathcal{E}^{\prime}(p)},
$$

where we have taken into account the symmetry of $\mathcal{E}$ under $p \mapsto 1-p$. From Eq. (2.25) it follows that $p=\mathrm{O}(\sqrt{\tau x})$ and

$$
\mathcal{E}^{\prime}(p)=\frac{p}{m(\alpha)}+\mathrm{O}\left(p^{3}\right)=\sqrt{\frac{2 \tau x}{m(\alpha)}}(1+\mathrm{O}(\tau x)) .
$$

Hence

$$
\frac{1}{|J|}\left(f-u_{0}\right)=-\gamma \sqrt{m(\alpha)} \tau^{3 / 2}+\mathrm{O}\left(\tau^{5 / 2}\right)
$$

where

$$
\gamma \equiv \sqrt{2} \int_{0}^{\infty} \frac{\log \left(1+\mathrm{e}^{-x}\right)}{\sqrt{x}} \mathrm{~d} x
$$

is a numeric constant which can be readily computed in closed form. Indeed,

$$
\begin{aligned}
\gamma & =\sqrt{2} \sum_{k=1}^{\infty} \frac{(-1)^{k+1}}{k} \int_{0}^{\infty} x^{-1 / 2} \mathrm{e}^{-k x} \mathrm{~d} x=\sqrt{2} \sum_{k=1}^{\infty} \frac{(-1)^{k+1}}{k^{3 / 2}} \int_{0}^{\infty} t^{-1 / 2} \mathrm{e}^{-t} \mathrm{~d} t \\
& =\sqrt{2 \pi} \sum_{k=1}^{\infty} \frac{(-1)^{k+1}}{k^{3 / 2}} \equiv \sqrt{2 \pi} \eta(3 / 2)
\end{aligned}
$$


where $\eta(z)$ denotes the Dirichlet's eta function. Using the well-know identity (cf. [36], Eq. (25.2.3))

$$
\eta(z)=\left(1-2^{1-z}\right) \zeta_{\mathrm{R}}(z)
$$

where $\zeta_{\mathrm{R}}$ denotes Riemann's zeta function, we finally obtain

$$
\gamma=(\sqrt{2}-1) \sqrt{\pi} \zeta_{\mathrm{R}}(3 / 2) \simeq 1.91794 .
$$

The asymptotic expansions of the remaining thermodynamic functions follow immediately from their definition and Eq. (3.7):

$$
\begin{aligned}
& \frac{1}{|J|}\left(u-u_{0}\right)=\frac{\gamma}{2} \sqrt{m(\alpha)} \tau^{3 / 2}+\mathrm{O}\left(\tau^{5 / 2}\right), \\
& \frac{c}{k_{\mathrm{B}}}=\frac{3 \gamma}{4} \sqrt{m(\alpha)} \tau^{1 / 2}+\mathrm{O}\left(\tau^{3 / 2}\right), \\
& \frac{s}{k_{\mathrm{B}}}=\frac{3 \gamma}{2} \sqrt{m(\alpha)} \tau^{1 / 2}+\mathrm{O}\left(\tau^{3 / 2}\right) .
\end{aligned}
$$

The low temperature behavior of the density of fermions can be computed in a similar way, with the result

$$
\frac{J}{|J|}\left(\nu_{\mathrm{F}}-\frac{1}{2}\right)+\frac{1}{2}=\gamma^{\prime} \sqrt{m(\alpha)} \tau^{1 / 2}+\mathrm{O}\left(\tau^{3 / 2}\right), \quad \gamma^{\prime} \equiv \sqrt{\pi}(\sqrt{2}-2) \zeta_{\mathrm{R}}\left(\frac{1}{2}\right) \simeq 1.51626 .
$$

Note that the previous formulas are valid for the limiting case $\alpha=0$, i.e., for the critical $X X$ model (2.23), whose effective mass is $m(0)=\left(8 \pi^{2}\right)^{-1}$. Thus, at low temperatures the $\mathrm{su}(1 \mid 1)$ elliptic chain $(2.1 a)-(2.20)$ is equivalent to the critical $X X$ model $(2.23)$ rescaled by the factor $\left(8 \pi^{2} m(\alpha)\right)^{-1}$. Similarly, replacing $\mathcal{E}(p)$ in Eqs. (3.2)-(3.5) by its $\alpha \rightarrow \infty$ limit $2 \pi^{2} p(1-p)$, we obtain the thermodynamic functions of the $\mathrm{su}(1 \mid 1)$ Haldane-Shastry chain. The resulting formulas exactly coincide with those deduced in Ref. [37] for its $\mathrm{su}(2)$ counterpart. Thus, in the thermodynamic limit the $\mathrm{su}(1 \mid 1)$ and $\mathrm{su}(2)$ Haldane-Shastry chains are equivalent (though this is certainly not the case for any finite value of $N$ ). Note, however, that the low temperature behavior of these models [37] markedly differs from that of the elliptic su(1|1) chain (cf. Fig. 3), since their dispersion relation is linear near $p=0$ and $p=1$.

\subsection{Level density}

One of the characteristic properties of the $\mathrm{su}(m)$ HS chain (and, indeed, of other related spin chains with long-range interactions) is the fact that in the limit $N \rightarrow \infty$ its level density (normalized to one) approaches a Gaussian distribution with parameters equal to the mean and the standard deviation of the spectrum [31]. By a suitable generalization of the central limit theorem, it was shown in Ref. [30] that this property also holds for systems with a factorizable partition function, i.e., such that

$$
\mathcal{Z}_{N}(T)=\prod_{l=0}^{N-1} \mathcal{Z}_{l}(T ; N)
$$



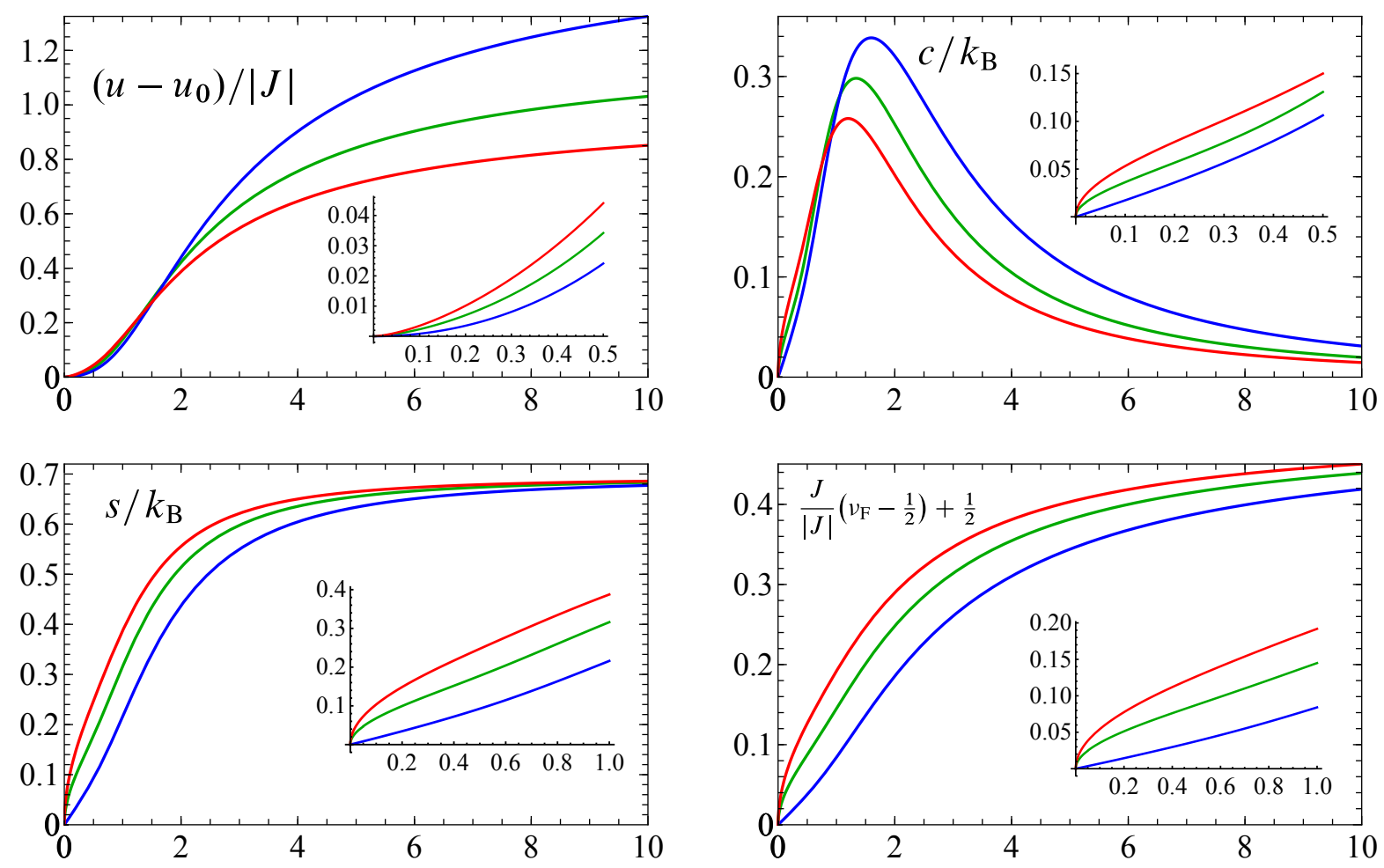

Figure 3. (Color online). Energy, specific heat, entropy (per site) and density of fermions for the elliptic su(1|1) chain with $\alpha=5$ (green), the critical $X X$ model (2.23) (red), and the $\mathrm{su}(1 \mid 1)$ Haldane-Shastry chain (blue) as a function of the dimensionless temperature $\tau=k_{\mathrm{B}} T /|J|$.

provided only that two general conditions are satisfied. The first of these conditions simply states that for sufficiently large $N$

$$
\sigma_{l} \leqslant C N^{-1 / 2} \sigma, \quad l=0, \ldots, N-1,
$$

for some positive constant $C$ independent of $N$. In the latter formula $\sigma_{l}$ and $\sigma$ respectively denote the standard deviation of the spectrum of the $l$-th subsystem and of the whole system, obviously related by

$$
\sigma^{2}=\sum_{l=0}^{N-1} \sigma_{l}^{2} .
$$

The second condition, which is of a rather more technical nature, is automatically satisfied when each $\mathcal{Z}_{l}$ is the partition function of a two-level system [30]. By Eq. (3.1), this is exactly what happens for the $\mathrm{su}(1 \mid 1)$ elliptic chain $(2.1 a)-(2.20)$, since in this case (3.8) holds with

$$
\mathcal{Z}_{l}=1+\mathrm{e}^{-\beta \mathcal{E}(l / N)} .
$$

(For simplicity, in the latter formula and for the rest of this section we have set $J=1$.) Moreover, from the latter equation we obviously have $\sigma_{l}=\frac{1}{2} \mathcal{E}(l / N)$, so that 
condition (3.9) reduces to

$$
\frac{1}{N} \sum_{k=0}^{N-1} \mathcal{E}(k / N)^{2} \geqslant C^{\prime} \mathcal{E}(l / N)^{2}, \quad l=0, \ldots, N-1
$$

for some positive constant $C^{\prime}$. These inequalities are clearly satisfied for $N$ large enough. Indeed, on the one hand we have

$$
\mathcal{E}(l / N) \leqslant \mathcal{E}_{\text {max }}, \quad l=0, \ldots, N-1,
$$

where $\mathcal{E}_{\max }$ is the maximum of $\mathcal{E}(p)$ in the compact interval [0,1], which is of course independent of $N$. (It can be shown that $\mathcal{E}_{\max }=\mathcal{E}(1 / 2)$, although this result is irrelevant for what follows.) On the other hand, since the LHS of Eq. (3.10) tends to $\int_{0}^{1} \mathcal{E}^{2}(p) \mathrm{d} p$ as $N \rightarrow \infty$, there exists a natural number $N_{0}$ such that

$$
\frac{1}{N} \sum_{k=0}^{N-1} \mathcal{E}(k / N)^{2} \geqslant \frac{1}{2} \int_{0}^{1} \mathcal{E}^{2}(p) \mathrm{d} p, \quad N \geqslant N_{0} .
$$

Thus condition (3.10) is satisfied for $N \geqslant N_{0}$ taking

$$
C^{\prime}=\frac{1}{2 \mathcal{E}_{\max }^{2}} \int_{0}^{1} \mathcal{E}^{2}(p) \mathrm{d} p .
$$

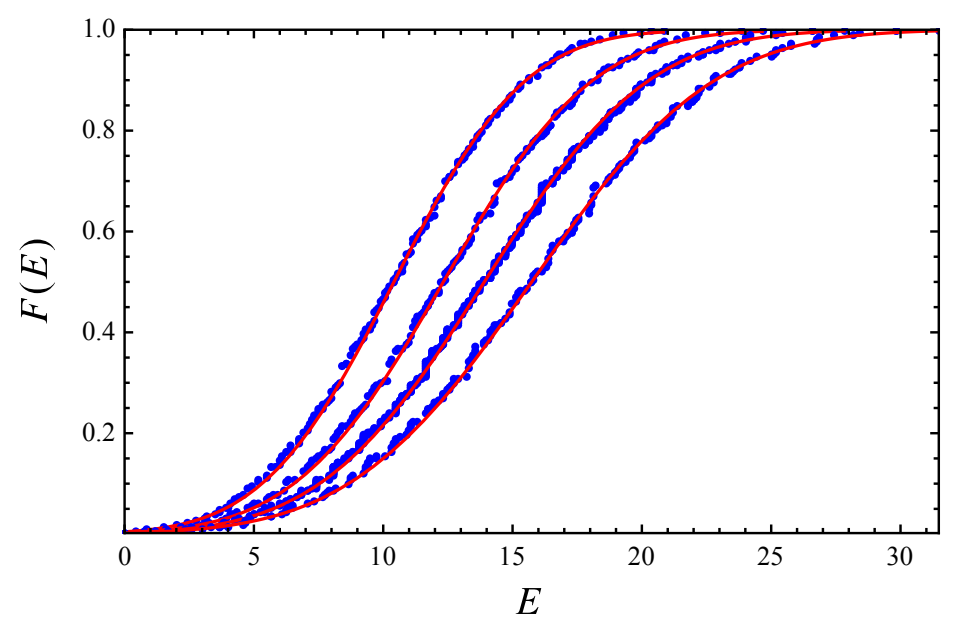

Figure 4. Blue dots: cumulative level density (3.11) of the $\mathrm{su}(1 \mid 1)$ elliptic chain for $N=10$ and $\alpha=2,5,10,50$ (left to right). Continuous red lines: corresponding cumulative Gaussian distributions (3.12) with parameters $\mu(\alpha)$ and $\sigma(\alpha)$ taken from the spectrum.

This proves that the level density of the elliptic su(1|1) chain becomes asymptotically Gaussian as the number of spins tends to infinity. In particular, this holds in the limiting cases $\alpha=0$ and $\alpha=\infty$, i.e., for the critical $X X$ model (2.23) and the $\operatorname{su}(1 \mid 1)$ Haldane-Shastry chain (1.1). In order to better illustrate this property, it is convenient to consider the (normalized) cumulative level density

$$
F(E)=2^{-N} \sum_{i ; E_{i} \leqslant E} d_{i}
$$


where $E_{1}<\cdots<E_{n}$ are the distinct eigenvalues and $d_{1}, \ldots, d_{n}$ their respective degeneracies. In Fig. 4 we have compared $F(E)$ for $N=10$ and several values of $\alpha$ with the corresponding cumulative Gaussian distribution

$$
G(E)=\frac{1}{2}\left[1+\operatorname{erf}\left(\frac{E-\mu(\alpha)}{\sqrt{2} \sigma(\alpha)}\right)\right]
$$

where $\mu(\alpha)$ and $\sigma(\alpha)$ respectively denote the mean and the standard deviation of the spectrum. It is apparent that, in spite of the relatively small value of $N$, the agreement between the exact and the approximate cumulative distributions is remarkable (in fact, for $N \gtrsim 15$ both distributions are virtually indistinguishable).

In view of the previous discussion, it is of interest to compute in closed form the mean and the standard deviation of the spectrum of the $\mathrm{su}(1 \mid 1)$ elliptic chain $(2.1 a)$ (2.20). In the first place, the mean energy $\mu$ of any translation-invariant chain of the form $(2.1 a)$ is easily calculated from Eq. (2.8), taking into account that

$$
\operatorname{tr}\left(a_{i}^{\dagger} a_{j}\right)=2^{N-1} \delta_{i j}
$$

Indeed,

$$
\mu=\langle H\rangle=2^{-N} \operatorname{tr} H=-\frac{N}{2} h(0)=\frac{N}{2} \sum_{l=1}^{N-1} h(l) .
$$

From Eq. (2.14), which can be equivalently rewritten as

$$
\sum_{j=1}^{N-1} \wp_{N}(j)=\frac{2}{\alpha^{2}}\left(N \hat{\eta}_{1}-\eta_{1}\right)
$$

and Eq. (2.20) we obtain the following explicit formula for the mean energy of the $\mathrm{su}(1 \mid 1)$ elliptic chain $(2.1 a)-(2.20)$ :

$$
\mu=\frac{N}{\pi^{2}} \sinh ^{2}(\pi / \alpha)\left(\hat{\eta}_{1}-\eta_{1}\right)
$$

The standard deviation of the spectrum of a translation-invariant chain (2.1a)-(2.6) can be computed in much the same way. Indeed, note to begin with that the trace of the product $a_{i}^{\dagger} a_{j} a_{k}^{\dagger} a_{l}$ vanishes for all $i, j, k, l$ except in the following three cases:

$$
\begin{aligned}
& \operatorname{tr}\left(a_{i}^{\dagger} a_{i} a_{i}^{\dagger} a_{i}\right)=\operatorname{tr}\left(a_{i}^{\dagger} a_{i}\right)=2^{N-1} \\
& \operatorname{tr}\left(a_{i}^{\dagger} a_{i} a_{j}^{\dagger} a_{j}\right)=2^{N-2} \quad(i \neq j) \\
& \operatorname{tr}\left(a_{i}^{\dagger} a_{j} a_{j}^{\dagger} a_{i}\right)=\operatorname{tr}\left(a_{i}^{\dagger} a_{i} a_{j} a_{j}^{\dagger}\right)=\operatorname{tr}\left(a_{i}^{\dagger} a_{i}\right)-\operatorname{tr}\left(a_{i}^{\dagger} a_{i} a_{j}^{\dagger} a_{j}\right)=2^{N-1}-2^{N-2}=2^{N-2} \quad(i \neq j) .
\end{aligned}
$$

Hence

$$
\begin{aligned}
\left\langle H^{2}\right\rangle & =2^{-N} \operatorname{tr}\left(H^{2}\right)=\sum_{i, j, k, l=1}^{N} h(|i-j|) h(|k-l|) \operatorname{tr}\left(a_{i}^{\dagger} a_{j} a_{k}^{\dagger} a_{l}\right) \\
& =\frac{N}{2} h(0)^{2}+\frac{1}{4} N(N-1) h(0)^{2}+\frac{1}{4} \sum_{1 \leqslant i \neq j \leqslant N} h(|i-j|)^{2} \\
& =\frac{1}{4} N(N+1)\left(\sum_{j=1}^{N-1} h(j)\right)^{2}+\frac{N}{4} \sum_{j=1}^{N-1} h(j)^{2},
\end{aligned}
$$


where we have used Eq. (2.7) with $h^{2}$ in place of $h$. We thus obtain

$$
\sigma^{2}=\left\langle H^{2}\right\rangle-\mu^{2}=\frac{N}{4}\left[\left(\sum_{j=1}^{N-1} h(j)\right)^{2}+\sum_{j=1}^{N-1} h(j)^{2}\right] .
$$

Using Eqs. (2.20) and (3.13), after a long but straightforward computation we arrive at the following formula for the variance of the energy of the elliptic chain $(2.1 a)-(2.20)$ :

$$
\sigma^{2}=N\left(\frac{\alpha}{\pi}\right)^{4} \sinh ^{4}(\pi / \alpha)\left[\frac{1}{4} \sum_{j=1}^{N-1} \wp_{N}^{2}(j)+\frac{1}{\alpha^{4}}\left(\eta_{1}^{2}-N \hat{\eta}_{1}^{2}\right)\right]
$$

The sum in the latter equation was evaluated in Ref. [28], with the result:

$$
\begin{aligned}
\sum_{j=1}^{N-1} \wp_{N}^{2}(j) & =\frac{1}{12}\left(N-\frac{6}{5}\right) g_{2}\left(\frac{N}{2}, \frac{\mathrm{i} \alpha}{2}\right)+\frac{1}{60} g_{2}\left(\frac{1}{2}, \frac{\mathrm{i} \alpha}{2}\right) \\
& =\frac{1}{12 \alpha^{4}}\left[\left(N-\frac{6}{5}\right) g_{2}\left(\frac{1}{2}, \frac{\mathrm{i} N}{2 \alpha}\right)+\frac{1}{5} g_{2}\left(\frac{1}{2}, \frac{\mathrm{i}}{2 \alpha}\right)\right],
\end{aligned}
$$

where we have used the homogeneity property of the Weierstrass invariant $g_{2}$ :

$$
g_{2}\left(\lambda \omega_{1}, \lambda \omega_{3}\right)=\frac{1}{\lambda^{4}} g_{2}\left(\omega_{1}, \omega_{3}\right) .
$$

Setting

$$
g_{2} \equiv g_{2}\left(\frac{1}{2}, \frac{\mathrm{i}}{2 \alpha}\right), \quad \hat{g}_{2} \equiv g_{2}\left(\frac{1}{2}, \frac{\mathrm{i} N}{2 \alpha}\right)
$$

we finally obtain the following exact formula for the variance of the spectrum of the chain $(2.1 a)-(2.20)$ :

$$
\sigma^{2}=\frac{N}{\pi^{4}} \sinh ^{4}(\pi / \alpha)\left[\left(N-\frac{6}{5}\right) \frac{\hat{g}_{2}}{48}+\frac{g_{2}}{240}+\eta_{1}^{2}-N \hat{\eta}_{1}^{2}\right] .
$$

To conclude this section, we shall compute the limiting values of $\mu / N$ and $\sigma^{2} / N$ when $N \rightarrow \infty$, which respectively coincide with the thermodynamic energy per particle $u$ and its derivative with respect to $\beta$ at $\beta=0$. The first of these limits is a straightforward consequence of Eq. (A.2) with $\omega_{1}=1 / 2$ and $q=\exp (-N \pi / \alpha) \rightarrow 0$ :

$$
\lim _{N \rightarrow \infty} \frac{\mu}{N}=\frac{1}{\pi^{2}} \sinh ^{2}(\pi / \alpha)\left(\frac{\pi^{2}}{6}-\eta_{1}\right) .
$$

Equating this expression to the value of $u$ at $\beta=0$ obtained from Eq. (3.3) with $J=1$, i.e.,

$$
u(0)=\frac{1}{2} \int_{0}^{1} \mathcal{E}(p) \mathrm{d} p
$$

we immediately obtain Eq. (3.6). When taking into account the definition $(2.21 b)$ of $\mathcal{E}$, the latter equation becomes the remarkable identity

$$
\int_{0}^{1}\left[\wp(p)-\left(\zeta(p)-2 \eta_{1} p\right)^{2}\right] \mathrm{d} p=\frac{2 \pi^{2}}{3} .
$$


Similarly, from Eq. (A.2) and the series

$$
g_{2}\left(\omega_{1}, \omega_{3}\right)=\left(\frac{\pi}{2 \omega_{1}}\right)^{4}\left(\frac{4}{3}+320 \sum_{n=1}^{\infty} \frac{n^{3} q^{2 n}}{1-q^{2 n}}\right), \quad q \equiv \exp \left(\mathrm{i} \pi \omega_{3} / \omega_{1}\right),
$$

(cf. [38]) we easily obtain

$$
\lim _{N \rightarrow \infty} \frac{\sigma^{2}}{N}=\frac{1}{\pi^{4}} \sinh ^{4}(\pi / \alpha)\left(\frac{g_{2}}{240}+\eta_{1}^{2}-\frac{\pi^{4}}{30}\right) .
$$

\section{The infinite chain}

We next consider the $\mathrm{su}(1 \mid 1)$ supersymmetric version of the infinite Inozemtsev chain [18], which we define as

$$
H=\frac{J}{2} \sum_{-\infty \leqslant j \neq k \leqslant \infty} g(j-k)\left(1-\mathscr{S}_{j k}\right),
$$

with

$$
g(x)=\left(\frac{\pi}{\alpha}\right)^{2} \sinh ^{-2}(\pi x / \alpha)
$$

This model is closely related to the $N \rightarrow \infty$ limit of the elliptic chain (2.1a)-(2.20). Indeed, if $h$ is defined by Eq. (2.20) and $x \neq 0$ is fixed, from Eqs. (A.4) (with $\omega_{1}=-\mathrm{i} \alpha / 2$, $\left.\omega_{3}=N / 2\right)$ and (A.2) (with $\omega_{1}=1 / 2, \omega_{3}=\mathrm{i} N /(2 \alpha)$ ) we easily obtain

$$
\lim _{N \rightarrow \infty} h(x)=\left(\frac{\alpha}{\pi}\right)^{2} \sinh ^{2}(\pi / \alpha) g(x) .
$$

Thus, it should be expected that the infinite chain (4.1a) is related to the thermodynamic limit of the finite elliptic chain $(2.1 a)-(2.20)$. In order to substantiate this heuristic observation, consider more generally a translation-invariant chain of the form $(4.1 a)$, defined by an arbitrary (smooth) even function $g$. Using Eq. (2.3) we can express the Hamiltonian of this chain in terms of fermion creation/annihilation operators $a_{n}, a_{n}^{\dagger}$ as

$$
H=-J \sum_{j, k=-\infty}^{\infty} g(j-k) a_{j}^{\dagger} a_{k}
$$

where

$$
g(0) \equiv-\sum_{0 \neq l \in \mathbb{Z}}^{\infty} g(l) .
$$

We next introduce the Fourier-transformed operators

$$
c(p)=\sum_{n=-\infty}^{\infty} \mathrm{e}^{-2 \pi \mathrm{i} n p} a_{n}
$$

where $p \in(0,1)$ is again the physical momentum $\S$ in units of $2 \pi$. From the identity

$$
\sum_{n=-\infty}^{\infty} \mathrm{e}^{2 \pi \mathrm{i} n x}=\delta(x)
$$

$\S$ Since the chain is invariant under integer translations, the momentum can be taken to belong to the "Brillouin interval" $[0,2 \pi)$. 
it easily follows that the operators $c(p)$ satisfy the canonical anticommutation relations

$$
\{c(p), c(q)\}=\left\{c^{\dagger}(p), c^{\dagger}(q)\right\}=0, \quad\left\{c^{\dagger}(p), c(q)\right\}=\delta(p-q) .
$$

Applying the inverse Fourier transform

$$
a_{n}=\int_{0}^{1} \mathrm{e}^{2 \pi \mathrm{in} n} c(p) \mathrm{d} p
$$

to the Hamiltonian (4.1) after a straightforward calculation we obtain

$$
H=J \int_{0}^{1} \varepsilon(p) c^{\dagger}(p) c(p) \mathrm{d} p,
$$

where the dispersion relation $\varepsilon(p)$ is given by

$$
\varepsilon(p)=\sum_{0 \neq j \in \mathbb{Z}}\left(1-\mathrm{e}^{ \pm 2 \pi \mathrm{i} j p}\right) g(j)=2 \sum_{j=1}^{\infty}[1-\cos (2 \pi j p)] g(j) .
$$

In particular, $H$ is completely integrable, since the operators $c^{\dagger}(p) c(p)$ are a commuting family of first integrals depending on the continuous parameter $p \in(0,1)$. Alternatively, from the definition of $c(p)$ it easily follows that the operators

$$
\sum_{n=-\infty}^{\infty} a_{n+k}^{\dagger} a_{n}, \quad k \in \mathbb{Z}
$$

form a denumerable family of commuting first integrals.

When $g$ is the function in Eq. (4.1b), the latter sum can be evaluated using similar techniques as in the finite (elliptic) case. Indeed, consider first the Fourier series

$$
\hat{g}(p) \equiv \sum_{0 \neq l \in \mathbb{Z}} \mathrm{e}^{-2 \pi \mathrm{i} l p} g(l), \quad 0<p<1 .
$$

In order to compute $\hat{g}$, we introduce the auxiliary function

$$
G(z)=\sum_{j=-\infty}^{\infty} \mathrm{e}^{-2 \pi \mathrm{i} j p} g(z+j)
$$

dependent on the parameter $p \in(0,1)$, which clearly satisfies

$$
G(z+1)=\mathrm{e}^{2 \pi \mathrm{i} p} G(z), \quad G(z+\mathrm{i} \alpha)=G(z)
$$

with $\exp (2 \pi \mathrm{i} p) \neq 1$. Each of the terms in Eq. (4.4) is analytic at the origin except the one with $j=0$, whose Laurent series about the origin is $g(z)=z^{-2}+\mathrm{O}(1)$. Thus $G(z)=z^{-2}+\mathrm{O}(1)$, and we can therefore apply Eqs. (B.2) and (B.5) with $f=G$ and $2 \omega_{3}=\mathrm{i} \alpha$. The Fourier series $\hat{g}(p)$ is now evaluated by computing the constant term in the Laurent series about the origin of $G(z)$ both directly and using Eq. (B.5). Indeed, since

$$
g(z)=\frac{1}{z^{2}}-\frac{\pi^{2}}{3 \alpha^{2}}+\mathrm{O}\left(z^{2}\right)
$$

and the terms with $j \neq 0$ in Eq. (4.4) are regular at the origin, the Laurent series of $G$ about the origin is given by

$$
G(z)=\frac{1}{z^{2}}-\frac{\pi^{2}}{3 \alpha^{2}}+\sum_{0 \neq j \in \mathbb{Z}} \mathrm{e}^{-2 \pi \mathrm{i} j p} g(j)+\mathrm{O}(z) \equiv \frac{1}{z^{2}}+\hat{g}(p)-\frac{\pi^{2}}{3 \alpha^{2}}+\mathrm{O}(z)
$$


Comparing with Eq. (B.5) we obtain

$$
\hat{g}(p)=\frac{1}{2} \wp_{1}(\mathrm{i} \alpha p)-\frac{1}{2}\left(\zeta_{1}(\mathrm{i} \alpha p)-2 \eta_{3} p\right)^{2}+\frac{\pi^{2}}{3 \alpha^{2}}, \quad 0<p<1 .
$$

The sum $\sum_{0 \neq l \in \mathbb{Z}} g(l)$ can now be computed by noting that, since the Fourier series defining $\hat{g}$ is uniformly convergent for all $p$ by Weierstrass's test, $\hat{g}$ is a continuous function of $p$. In particular

$$
\sum_{0 \neq l \in \mathbb{Z}} g(l)=\lim _{p \rightarrow 0} \hat{g}(p)=\frac{2 \eta_{3}}{\mathrm{i} \alpha}+\frac{\pi^{2}}{3 \alpha^{2}},
$$

where the limit has been computed using Eqs. (2.15) and (B.4). Substituting Eqs. (4.5) and (4.6) into Eq. (4.3) we finally obtain

$$
\varepsilon(p)=\frac{1}{2}\left(\zeta_{1}(\mathrm{i} \alpha p)-2 \eta_{3} p\right)^{2}-\frac{1}{2} \wp_{1}(\mathrm{i} \alpha p)+\frac{2 \eta_{3}}{\mathrm{i} \alpha},
$$

or, taking into account the homogeneity property (2.18) of the Weierstrass functions,

$$
\varepsilon(p)=\frac{1}{2 \alpha^{2}}\left[\wp(p)-\left(\zeta(p)-2 \eta_{1} p\right)^{2}-4 \eta_{1}\right] \equiv\left(\frac{\pi}{\alpha}\right)^{2} \sinh ^{-2}\left(\frac{\pi}{\alpha}\right) \mathcal{E}(p),
$$

where $\mathcal{E}(p)$ is the dispersion relation of the (finite) elliptic chain (cf. Eq. (2.21b)). Thus the infinite hyperbolic chain $(4.1 a)$ turns out to be equivalent to the thermodynamic limit of the elliptic $\mathrm{su}(1 \mid 1)$ chain $(2.1 a)$-(2.20) (up to a constant factor).

Remark 6. The dispersion relation (4.7) of the su(1|1) infinite chain (4.1) coincides with the expression for the energy of the 1-magnons with momentum $2 \pi p(\bmod 2 \pi)$ of its $\mathrm{su}(2)$ counterpart which can be found in Ref. [27]. This is no coincidence since, as in the finite case, it can be shown that the same is true for any translation-invariant chain of the form $(4.1 a)$.

Remark 7. The sum (4.6) is of interest in the context of the AdS/CFT conjecture. Indeed, in Ref. [25] it is shown that the perturbative expansion of the (planar, two complex scalar fields) dilation operator of $\mathcal{N}=4$ super Yang-Mills theory up to three loops can be obtained from the spectrum of the $\mathrm{su}(2)$ analog of the infinite hyperbolic chain $(4.1 a)$

$$
H=\frac{1}{8} \sum_{-\infty \leqslant j \neq l \leqslant \infty} \sinh ^{-2}(\kappa(j-l))\left(1-S_{j l}\right)
$$

provided that the Yang-Mills coupling constant $\lambda$ is related to the parameter $\kappa$ by

$$
\lambda=4 \pi^{2} \sum_{n=1}^{\infty} \sinh ^{-2}(n \kappa)
$$

(cf. [25], Eq. (2.10)). Using Eq. (4.6) with $\alpha=\pi / \kappa$ we easily arrive at the following explicit formula for the coupling constant $\lambda$ :

$$
\lambda=\frac{4 \pi^{2}}{\kappa^{2}}\left[\frac{\kappa}{\mathrm{i} \pi} \eta_{3}\left(\frac{1}{2}, \frac{\mathrm{i} \pi}{2 \kappa}\right)+\frac{\kappa^{2}}{6}\right]=\frac{2 \pi^{2}}{3}-4 \eta_{1}\left(\frac{1}{2}, \frac{\mathrm{i} \kappa}{2 \pi}\right) .
$$


From Eqs. (A.2)-(A.3) with $\omega_{1}=1 / 2, \omega_{3}=i \kappa /(2 \pi)$ we obtain the expansion

$$
\lambda=16 \pi^{2} \sum_{n=1}^{\infty} \frac{n \mathrm{e}^{-2 n \kappa}}{1-\mathrm{e}^{-2 n \kappa}}=16 \pi^{2} \sum_{n=1}^{\infty} \sigma_{1}(n) \mathrm{e}^{-2 n \kappa},
$$

where $\sigma_{1}(n)$ denotes the number-theoretic divisor function

$$
\sigma_{1}(n) \equiv \sum_{j \text { divides } n} j
$$

\section{Conclusions and outlook}

We have introduced the $\mathrm{su}(1 \mid 1)$ supersymmetric version of Inozemtsev's elliptic (finite) and hyperbolic (infinite) spin chains, presented a proof of their integrability, and obtained their exact solution. This is rather unexpected, in view of the fact that no rigorous proof of the integrability or complete solution of the apparently simpler $\operatorname{su}(2)$ version of these models is known. Taking advantage of the explicit knowledge of the spectrum, we have been able to compute in closed form the thermodynamic functions, showing that at low temperatures the $\mathrm{su}(1 \mid 1)$ elliptic chain is essentially equivalent to a critical $X X$ model. We have also rigorously proved that the spectrum is normally distributed in the thermodynamic limit, as is typically the case with spin chains of Haldane-Shastry type. In fact, these results also apply to the standard $X X$ model at a critical value of the magnetic field, since this model is obtained from su(1|1) elliptic chain when the imaginary period of the interaction strength tends to zero.

Our results suggest several new developments and open problems. In the first place, it would be of interest to determine whether the elliptic $\mathrm{su}(1 \mid 1)$ and $\mathrm{su}(2)$ chains are equivalent in the thermodynamic limit, as we have shown to be the case for their Haldane-Shastry counterparts. Another natural problem is the extension of the present work to elliptic chains of $\mathrm{su}(m \mid n)$ type. Note, in this respect, that in the thermodynamic limit and at low temperature the $\mathrm{su}(m \mid 1)$ Haldane-Shastry chain is equivalent to a model of $m$ species of non-interacting fermions, with the same dispersion relation as its $\mathrm{su}(2)$ and $\mathrm{su}(1 \mid 1)$ versions [29]. A third line of future research opened up by our exact solution of the $\mathrm{su}(1 \mid 1)$ elliptic chain is the study of properties of its spectrum of relevance in the characterization of integrability vs. quantum chaos (nearest-neighbor spacing distribution, spectral noise, etc.), as has been done for spin chains of HaldaneShastry type (see, e.g., Ref. [6,32,39,40]). Finally, another potential application of our results is the computation of the entanglement entropy of the ground state of the elliptic chain (2.1a)-(2.20), using the method outlined in Ref. [41] for the $X X$ model in a constant magnetic field.

\section{Acknowledgments}

This work was supported in part by Spain's MINECO under grant no. FIS2011-22566. 


\section{Appendix A. Evaluation of some limits involving the Weierstrass elliptic function}

Our starting point shall be the two trigonometric series

$$
\begin{aligned}
& \wp\left(z ; \omega_{1}, \omega_{3}\right)=-\frac{\eta_{1}\left(\omega_{1}, \omega_{3}\right)}{\omega_{1}}+\frac{\pi^{2}}{4 \omega_{1}^{2}} \sin ^{-2}\left(\frac{\pi z}{2 \omega_{1}}\right)-\frac{2 \pi^{2}}{\omega_{1}^{2}} \sum_{n=1}^{\infty} \frac{n q^{2 n}}{1-q^{2 n}} \cos \left(\frac{n \pi z}{\omega_{1}}\right) \\
& \eta_{1}\left(\omega_{1}, \omega_{3}\right)=\frac{\pi^{2}}{12 \omega_{1}}-\frac{2 \pi^{2}}{\omega_{1}} \sum_{n=1}^{\infty} \frac{q^{2 n}}{\left(1-q^{2 n}\right)^{2}},
\end{aligned}
$$

where

$$
q \equiv \mathrm{e}^{\mathrm{i} \pi \omega_{3} / \omega_{1}}
$$

$\left|\operatorname{Im}\left(z / \omega_{1}\right)\right|<2 \operatorname{Im}\left(\omega_{3} / \omega_{1}\right)$, and $z \neq m \omega_{1}+n \omega_{3}$ for all $m, n \in \mathbb{Z}$ (see [36], Eqs. (23.8.1)$(23-8.5))$. Both series can be combined by noting that

$$
\sum_{n=1}^{\infty} \frac{q^{2 n}}{\left(1-q^{2 n}\right)^{2}}=\sum_{n=1}^{\infty} \sum_{m=1}^{\infty} m q^{2 m n}=\sum_{m, n=1}^{\infty} n q^{2 m n}=\sum_{n=1}^{\infty} n \sum_{m=1}^{\infty} q^{2 m n}=\sum_{n=1}^{\infty} \frac{n q^{2 n}}{1-q^{2 n}},
$$

where all the rearrangements are justified by the absolute convergence of the double series (note that $|q|<1$ on account of the condition $\operatorname{Im}\left(\omega_{3} / \omega_{1}\right)>0$ ). Inserting (A.2) into (A.1) we thus obtain

$$
\wp\left(z ; \omega_{1}, \omega_{3}\right)=\frac{\pi^{2}}{\omega_{1}^{2}}\left[-\frac{1}{12}+\frac{1}{4} \sin ^{-2}\left(\frac{\pi z}{2 \omega_{1}}\right)+4 \sum_{n=1}^{\infty} \frac{n q^{2 n}}{1-q^{2 n}} \sin ^{2}\left(\frac{n \pi z}{2 \omega_{1}}\right)\right] .
$$

Equation (1.3) immediately follows by taking $\omega_{1}=N / 2, \omega_{3}=\mathrm{i} \alpha / 2$ and letting $\alpha \rightarrow \infty$, i.e., $q=\exp (-\pi \alpha / N) \rightarrow 0$. In order to examine the behavior of $\wp_{N}(x)$ as $\alpha \rightarrow 0$, we first note that

$$
\wp_{N}(x) \equiv \wp\left(x ; \frac{N}{2}, \frac{\mathrm{i} \alpha}{2}\right)=\wp\left(x ;-\frac{\mathrm{i} \alpha}{2}, \frac{N}{2}\right) .
$$

If $1 \leqslant x \leqslant N-1$, from Eq. (A.4) with $\omega_{1}=-\mathrm{i} \alpha / 2, \omega_{3}=N / 2$, and thus $q=\exp (-N \pi / \alpha) \rightarrow 0$, we have

$$
\begin{aligned}
\mathrm{e}^{2 \pi / \alpha}\left(\frac{\alpha^{2}}{4 \pi^{2}} \wp_{N}(x)-\frac{1}{12}\right)= & \frac{q^{2(x-1) / N}}{\left(1-q^{2 x / N}\right)^{2}}+\sum_{n=1}^{\infty} \frac{n q^{2[n(N-x)-1] / N}\left(1-q^{2 n x / N}\right)^{2}}{1-q^{2 n}} \\
& \underset{q \rightarrow 0}{\longrightarrow} \delta_{1, x}+\delta_{N-1, x} .
\end{aligned}
$$

We shall next prove that the Hamiltonian (2.1a)-(2.20) smoothly interpolates between the Heisenberg and the Haldane-Shastry Hamiltonians as the parameter $\alpha$ ranges from zero to infinity. In other words, if $h(x)$ denotes the function defined by Eq. (2.20) we shall show that

$$
\lim _{\alpha \rightarrow 0} h(x)=\delta_{1, x}+\delta_{N-1, x}, \quad \lim _{\alpha \rightarrow \infty} h(x)=\frac{\pi^{2}}{N^{2}} \sin ^{-2}\left(\frac{\pi x}{N}\right),
$$

where in the former limit $1 \leqslant x \leqslant N-1$. Consider, to begin with, the limit $\alpha \rightarrow 0$. From Eq. (A.2) with $\omega_{1}=1 / 2, \omega_{3}=\mathrm{i} N /(2 \alpha)$ and, therefore, $q=\exp (-N \pi / \alpha) \rightarrow 0$ we have

$$
\hat{\eta}_{1} \equiv \eta_{1}\left(\frac{1}{2}, \frac{\mathrm{i} N}{2 \alpha}\right)=\frac{\pi^{2}}{6}+\mathrm{O}\left(\mathrm{e}^{-2 N \pi / \alpha}\right)
$$


Thus

$$
\begin{aligned}
h(x) & =\left(\frac{\alpha}{\pi}\right)^{2} \sinh ^{2}(\pi / \alpha)\left(\wp_{N}(x)-\frac{\pi^{2}}{3 \alpha^{2}}\right)+\mathrm{O}\left(\mathrm{e}^{-2 \pi(N-1) / \alpha}\right) \\
& =\left(1-\mathrm{e}^{-2 \pi / \alpha}\right)^{2} \cdot \mathrm{e}^{2 \pi / \alpha}\left(\frac{\alpha^{2}}{4 \pi^{2}} \wp_{N}(x)-\frac{1}{12}\right)+\mathrm{O}\left(\mathrm{e}^{-2 \pi(N-1) / \alpha}\right),
\end{aligned}
$$

which indeed tends to $\delta_{1, x}+\delta_{N-1, x}$ for $1 \leqslant x \leqslant N-1$ as $\alpha \rightarrow 0$ on account of Eq. (A.5).

Consider now the $\alpha \rightarrow \infty$ limit. From the homogeneity property of the Weierstrass zeta function (see Eq. (2.18)) we have

$$
\hat{\eta}_{1}=\frac{\mathrm{i} \alpha}{N} \eta_{3}\left(\frac{1}{2}, \frac{\mathrm{i} \alpha}{2 N}\right)=\frac{\alpha}{N}\left[\pi-\frac{\alpha}{N} \eta_{1}\left(\frac{1}{2}, \frac{\mathrm{i} \alpha}{2 N}\right)\right],
$$

where in the last step we have used Legendre's relation (cf. [36], Eq. (23.2.14))

$$
\omega_{3} \eta_{1}\left(\omega_{1}, \omega_{3}\right)-\omega_{1} \eta_{3}\left(\omega_{1}, \omega_{3}\right)=\frac{\mathrm{i} \pi}{2} .
$$

From the series (A.2) we easily obtain

$$
\lim _{\alpha \rightarrow \infty} \eta_{1}\left(\frac{1}{2}, \frac{\mathrm{i} \alpha}{2 N}\right)=\frac{\pi^{2}}{6}
$$

and therefore

$$
h(x)=\left(\frac{\alpha}{\pi}\right)^{2} \sinh ^{2}(\pi / \alpha)\left(\wp_{N}(x)+\frac{2}{N^{2}} \eta_{1}\left(\frac{1}{2}, \frac{\mathrm{i} \alpha}{2 N}\right)-\frac{2 \pi}{N \alpha}\right) \underset{\alpha \rightarrow \infty}{\longrightarrow} \frac{\pi^{2}}{N^{2}} \sin ^{-2}\left(\frac{\pi x}{N}\right)
$$

on account of Eq. (1.3).

We shall finally prove that the dispersion relation $(2.21 b)$ of the elliptic $\mathrm{su}(1 \mid 1)$ chain (2.1a)-(2.20) does tend to the dispersion relations of the critical $X X$ model (2.23) and the $\mathrm{su}(1 \mid 1)$ Haldane-Shastry chain (1.1) respectively as $\alpha \rightarrow 0$ and $\alpha \rightarrow \infty$. In other words, we shall show that

$$
\lim _{\alpha \rightarrow 0} \mathcal{E}(p)=4 \sin ^{2}(\pi p), \quad \lim _{\alpha \rightarrow \infty} \mathcal{E}(p)=2 \pi^{2} p(1-p)
$$

(cf. Eqs. (2.22)-(2.24)). Consider, to begin with, the former of these limits. Using Eqs. (A.2)-(A.4) with $\omega_{1}=1 / 2, \omega_{3}=\mathrm{i} \alpha / 2$ and, hence, $q=\exp (-\pi / \alpha) \rightarrow 0$ we readily obtain

$$
\wp(p)-4 \eta_{1}=\pi^{2} \cot ^{2}(\pi p)+16 \pi^{2} \mathrm{e}^{-2 \pi / \alpha}\left(1+\sin ^{2}(\pi p)\right)+\mathrm{O}\left(\mathrm{e}^{-4 \pi / \alpha}\right) .
$$

Similarly, from the series

$$
\zeta\left(z ; \omega_{1}, \omega_{3}\right)=\frac{\eta_{1}\left(\omega_{1}, \omega_{3}\right)}{\omega_{1}} z+\frac{\pi}{2 \omega_{1}} \cot \left(\frac{\pi z}{2 \omega_{1}}\right)+\frac{2 \pi}{\omega_{1}} \sum_{n=1}^{\infty} \frac{q^{2 n}}{1-q^{2 n}} \sin \left(\frac{n \pi z}{\omega_{1}}\right),
$$

valid for $\left|\operatorname{Im}\left(z / \omega_{1}\right)\right|<2 \operatorname{Im}\left(\omega_{3} / \omega_{1}\right)$ and $z \neq m \omega_{1}+n \omega_{3}$ for all $m, n \in \mathbb{Z}$ (cf [36], Eq. (23.8.2)) we have

$$
\zeta(p)-2 \eta_{1} p=\pi \cot (\pi p)+4 \pi \mathrm{e}^{-2 \pi / \alpha} \sin (2 \pi p)+\mathrm{O}\left(\mathrm{e}^{-4 \pi / \alpha}\right) .
$$

Hence

$$
\wp(p)-\left(\zeta(p)-2 \eta_{1} p\right)^{2}-4 \eta_{1}=32 \pi^{2} \mathrm{e}^{-2 \pi / \alpha} \sin ^{2}(\pi p)+\mathrm{O}\left(\mathrm{e}^{-4 \pi / \alpha}\right)
$$


and multiplying by the factor $\sinh ^{2}(\pi / \alpha) /\left(2 \pi^{2}\right)$ we easily obtain

$$
\mathcal{E}(p)=4 \sin ^{2}(\pi p)+\mathrm{O}\left(\mathrm{e}^{-2 \pi / \alpha}\right) \underset{\alpha \rightarrow 0}{\longrightarrow} 4 \sin ^{2}(\pi p) .
$$

Consider, finally, the $\alpha \rightarrow \infty$ limit. Using the homogeneity relations (2.18) we can write

$\mathcal{E}(p)=\frac{1}{2}\left(\frac{\alpha}{\pi}\right)^{2} \sinh ^{2}(\pi / \alpha)\left[\left(\zeta_{1}(\mathrm{i} \alpha p)-2 \eta_{3}\left(\frac{1}{2}, \frac{\mathrm{i} \alpha}{2}\right) p\right)^{2}-\wp_{1}(\mathrm{i} \alpha p)+\frac{4}{\mathrm{i} \alpha} \eta_{3}\left(\frac{1}{2}, \frac{\mathrm{i} \alpha}{2}\right)\right]$,

where $\wp_{1}$ and $\zeta_{1}$ denote the Weierstrass functions with periods 1 and $i \alpha$. From Eqs. (A.2), (A.4) and (A.7) with $\omega_{1}=1 / 2, \omega_{3}=\mathrm{i} \alpha / 2$, so that $q=\exp (-\pi \alpha) \rightarrow 0$, and the Legendre relation (A.6), we now obtain

$$
\begin{aligned}
& \wp_{1}(\mathrm{i} \alpha p)=-\frac{\pi^{2}}{3}+\mathrm{O}\left(\mathrm{e}^{-2 \pi \alpha \min (p, 1-p)}\right) \\
& \begin{aligned}
& \zeta_{1}(\mathrm{i} \alpha p)-2 \eta_{3}\left(\frac{1}{2}, \frac{\mathrm{i} \alpha}{2}\right) p=2 \pi \mathrm{i} p-\mathrm{i} \pi \operatorname{coth}(\pi \alpha p)+\mathrm{O}\left(\mathrm{e}^{-2 \pi \alpha(1-p)}\right) \\
&=\pi \mathrm{i}(2 p-1)+\mathrm{O}\left(\mathrm{e}^{-2 \pi \alpha \min (p, 1-p)}\right) \\
& \frac{4}{\mathrm{i} \alpha} \eta_{3}\left(\frac{1}{2}, \frac{\mathrm{i} \alpha}{2}\right)=4 \eta_{1}\left(\frac{1}{2}, \frac{\mathrm{i} \alpha}{2}\right)-\frac{4 \pi}{\alpha}=\frac{2 \pi^{2}}{3}+\mathrm{O}\left(\alpha^{-1}\right) .
\end{aligned}
\end{aligned}
$$

These asymptotic relations and Eq. (A.9) immediately lead to

$\mathcal{E}(p)=\frac{\pi^{2}}{2}\left[1-(2 p-1)^{2}\right]+\mathrm{O}\left(\alpha^{-1}\right)=2 \pi^{2} p(1-p)+\mathrm{O}\left(\alpha^{-1}\right) \underset{\alpha \rightarrow \infty}{\longrightarrow} 2 \pi^{2} p(1-p)$,

as claimed. Note that the convergence of $\mathcal{E}(p)$ to the Haldane-Shastry dispersion relation (2.22) as $\alpha \rightarrow \infty$ is much slower than its convergence to the $X X$ dispersion relation (2.24) as $\alpha \rightarrow 0$, as can be seen from Eqs. (A.8)-(A.10) (and is also apparent from Fig. 2).

\section{Appendix B. Quasi-periodic functions}

In this section we derive several key results on quasi-periodic functions needed for the explicit evaluation of the dispersion relation of the elliptic chain (2.1a)-(2.20) and its infinite (hyperbolic) counterpart (4.1a)-(4.1b).

By definition, a function $f(z)$ is (strictly) quasi-periodic with half-periods $\omega_{1}$ and $\omega_{3}$ provided that

$$
f\left(z+2 \omega_{1}\right)=\mathrm{e}^{2 \pi \mathrm{i} p} f(z), \quad f\left(z+2 \omega_{3}\right)=f(z),
$$

where $p \notin \mathbb{Z}$ is a real constant. Since the periods are assumed to be independent, i.e., $\operatorname{Im}\left(\omega_{3} / \omega_{1}\right) \neq 0$, from now on we shall suppose without loss of generality that $\operatorname{Im}\left(\omega_{3} / \omega_{1}\right)>0$. One of the most basic results about quasi-periodic functions is the following immediate consequence of Liouville's theorem in analytic function theory: if a quasi-periodic function $f$ is analytic in the closed period parallelogram

$$
\pi_{\omega_{1}, \omega_{3}}=\left\{2 x \omega_{1}+2 y \omega_{3} \mid 0 \leqslant x, y \leqslant 1\right\},
$$

then it is identically zero. Indeed, by the analiticity hypothesis $f$ is bounded in the compact set $\pi_{\omega_{1}, \omega_{3}}$. The quasi-periodicity conditions then imply that $f$ is entire 
and bounded, since for every $z \in \mathbb{C}$ we can always find two integers $m, n$ such that $z-2 m \omega_{1}-2 n \omega_{3}=z_{0} \in \pi_{\omega_{1}, \omega_{3}}$, and hence

$$
|f(z)|=\left|\mathrm{e}^{2 \pi \mathrm{i} m p} f\left(z_{0}\right)\right|=\left|f\left(z_{0}\right)\right| .
$$

By Liouville's theorem, $f$ reduces to a constant $c$, which must vanish on account of the conditions $c=\mathrm{e}^{2 \pi \mathrm{i} p} c$ and $p \notin \mathbb{Z}$.

The previous result implies that, just as elliptic (doubly periodic) functions, quasiperiodic functions are essentially determined by their singularities. We shall only need here a very simple application of this idea, which we shall discuss next. More precisely, suppose that $f$ is a quasi-periodic function whose only singularities are double poles on the period lattice $2 m \omega_{1}+2 n \omega_{3}(m, n \in \mathbb{Z})$, and whose Laurent series about the origin is

$$
f(z)=\frac{1}{z^{2}}+\mathrm{O}(1)
$$

Let us assume, for simplicity, that $2 \omega_{1}=1$ (otherwise, it suffices to replace $f(z)$ by $f\left(\omega_{1} z\right)$ and $\omega_{3}$ by $\omega_{3} / \omega_{1}$ in what follows). We shall then show that

$$
\begin{aligned}
f(z)=\frac{\sigma_{1}\left(\omega_{3} p+z\right)}{\sigma_{1}\left(\omega_{3} p-z\right)} \mathrm{e}^{-A(p) z}[ & \wp_{1}(z)-\wp_{1}\left(\omega_{3} p\right) \\
& \left.+B(p)\left(\zeta_{1}(z)-\zeta_{1}\left(z+\omega_{3} p\right)+\zeta_{1}\left(2 \omega_{3} p\right)-\zeta_{1}\left(\omega_{3} p\right)\right)\right]
\end{aligned}
$$

with

$$
A(p)=2 \eta_{3}\left(1 / 2, \omega_{3}\right) p \equiv 2 \eta_{3} p, \quad B(p)=2\left(\eta_{3} p-\zeta_{1}\left(\omega_{3} p\right)\right)
$$

Here

$\wp_{1}(z) \equiv \wp\left(z ; 1 / 2, \omega_{3}\right), \quad \zeta_{1}(z) \equiv \zeta\left(z ; 1 / 2, \omega_{3}\right), \quad \sigma_{1}(z) \equiv \sigma\left(z ; 1 / 2, \omega_{3}\right)$,

where $\sigma\left(z ; \omega_{1}, \omega_{3}\right)$ denotes the Weierstrass sigma function [19]. Indeed, let us denote by $f_{0}$ the RHS of Eq. (B.2). The term in square brackets in $f_{0}$ is clearly periodic, with periods 1 and $2 \omega_{3}$, due to the identities

$$
\zeta\left(z+2 \omega_{i} ; \omega_{1}, \omega_{3}\right)=\zeta\left(z ; \omega_{1}, \omega_{3}\right)+2 \eta_{i}\left(\omega_{1}, \omega_{3}\right) .
$$

On the other hand, from the relation

$$
\sigma\left(z+2 \omega_{i} ; \omega_{1}, \omega_{3}\right)=-\exp \left[2 \eta_{i}\left(\omega_{1}, \omega_{3}\right)\left(z+\omega_{i}\right)\right] \sigma\left(z ; \omega_{1}, \omega_{3}\right)
$$

and Legendre's identity (A.6) it follows that the factor

$$
g(z) \equiv \frac{\sigma_{1}\left(\omega_{3} p+z\right)}{\sigma_{1}\left(\omega_{3} p-z\right)} \mathrm{e}^{-A(p) z}
$$

satisfies

$$
g(z+1)=\mathrm{e}^{2 \pi \mathrm{i} p} g(z), \quad g\left(z+2 \omega_{3}\right)=g(z) .
$$

Thus $f_{0}$ is quasi-periodic, with the same half-periods as $f$. Furthermore, $f_{0}$ is analytic on the whole complex plane except on the period lattice. Indeed, the simple zero of $\sigma_{1}\left(\omega_{3} p-z\right)$ at $\omega_{3} p$ (and congruent points) is canceled by the zero of the term in square brackets, while the simple pole of the latter term due to $\zeta_{1}\left(z+\omega_{3} p\right)$ at $z=-\omega_{3} p$ (and 
points congruent to it) is canceled by the simple zero of $\sigma_{1}\left(z+\omega_{3} p\right)$. The behavior at the origin of $f_{0}$ can be easily determined using the relation

$$
\zeta\left(z ; \omega_{1}, \omega_{3}\right)=\frac{\sigma^{\prime}\left(z ; \omega_{1}, \omega_{3}\right)}{\sigma\left(z ; \omega_{1}, \omega_{3}\right)}=\frac{1}{z}+\mathrm{O}\left(z^{3}\right)
$$

from which it follows that

$$
\begin{aligned}
\frac{\sigma_{1}\left(\omega_{3} p+z\right)}{\sigma_{1}\left(\omega_{3} p-z\right)} & =\frac{\sigma_{1}\left(\omega_{3} p\right)+\sigma_{1}^{\prime}\left(\omega_{3} p\right) z+\mathrm{O}\left(z^{2}\right)}{\sigma_{1}\left(\omega_{3} p\right)-\sigma_{1}^{\prime}\left(\omega_{3} p\right) z+\mathrm{O}\left(z^{2}\right)}=\frac{1+\zeta_{1}\left(\omega_{3} p\right) z+\mathrm{O}\left(z^{2}\right)}{1-\zeta_{1}\left(\omega_{3} p\right) z+\mathrm{O}\left(z^{2}\right)} \\
& =1+2 \zeta_{1}\left(\omega_{3} p\right) z+\mathrm{O}\left(z^{2}\right) .
\end{aligned}
$$

Using this identity it is straightforward to derive the principal part of $f_{0}$ at the origin, namely

$$
f_{0}(z)=\frac{1}{z^{2}}+\frac{B(p)-A(p)+2 \zeta_{1}\left(\omega_{3} p\right)}{z}+\mathrm{O}(1) .
$$

Since the coefficient of $1 / z$ in the latter series vanishes identically on account of the definitions of $A(p)$ and $B(p)$, both sides of (B.2) have the same principal part at the origin and hence, by quasi-periodicity, at all points congruent to it. Hence the difference $f-f_{0}$ is strictly quasi-periodic and entire, and therefore vanishes identically by the basic property of quasi-periodic functions proved at the beginning of this appendix.

The last result needed for the evaluation of the dispersion relations of the elliptic and hyperbolic su(1|1) chains is the following explicit formula for the constant term in the Laurent expansion about the origin of the function $f(z)$ in Eq. (B.2):

$$
\lim _{z \rightarrow 0}\left(f(z)-\frac{1}{z^{2}}\right)=\frac{1}{2} \wp_{1}\left(2 \omega_{3} p\right)-\frac{1}{2}\left(\zeta_{1}\left(2 \omega_{3} p\right)-2 \eta_{3} p\right)^{2}
$$

To prove this formula, note that

$$
\frac{\sigma_{1}\left(\omega_{3} p+z\right)}{\sigma_{1}\left(\omega_{3} p-z\right)}=1+2 \zeta_{1}\left(\omega_{3} p\right) z+2 \zeta_{1}\left(\omega_{3} p\right)^{2} z^{2}+\mathrm{O}\left(z^{3}\right)
$$

from which it easily follows that

$$
\mathrm{e}^{-A(p) z} \frac{\sigma_{1}\left(\omega_{3} p+z\right)}{\sigma_{1}\left(\omega_{3} p-z\right)}=1-B(p) z+\frac{1}{2} B(p)^{2} z^{2}+\mathrm{O}\left(z^{3}\right)
$$

Using this expansion and the Laurent series about the origin of the term in square brackets in Eq. (B.2), namely

$$
\frac{1}{z^{2}}+\frac{B(p)}{z}+B(p)\left[\zeta_{1}\left(2 \omega_{3} p\right)-2 \zeta_{1}\left(\omega_{3} p\right)\right]-\wp_{1}\left(\omega_{3} p\right)+\mathrm{O}(z),
$$

we readily obtain

$$
f(z)=\frac{1}{z^{2}}+B(p)\left[\zeta_{1}\left(2 \omega_{3} p\right)-2 \zeta_{1}\left(\omega_{3} p\right)\right]-\wp_{1}\left(\omega_{3} p\right)-\frac{1}{2} B(p)^{2}+\mathrm{O}(z) .
$$

We thus have

$$
\begin{aligned}
\lim _{z \rightarrow 0}\left(f(z)-\frac{1}{z^{2}}\right) & =B(p)\left(\zeta_{1}\left(2 \omega_{3} p\right)-2 \zeta_{1}\left(\omega_{3} p\right)\right)-\wp_{1}\left(\omega_{3} p\right)-\frac{1}{2} B(p)^{2} \\
& =2 \zeta_{1}\left(2 \omega_{3} p\right)\left(\eta_{3} p-\zeta_{1}\left(\omega_{3} p\right)\right)+2 \zeta_{1}\left(\omega_{3} p\right)^{2}-2 \eta_{3}^{2} p^{2}-\wp_{1}\left(\omega_{3} p\right),
\end{aligned}
$$


where we have used the definition (B.3) of $B(p)$. Equation (B.5) now follows straightforwardly from the duplication formulas

$$
\wp(z)=-\frac{1}{2} \wp(2 z)+\frac{1}{8}\left(\frac{\wp^{\prime \prime}(z)}{\wp^{\prime}(z)}\right)^{2}, \quad \zeta(z)=\frac{1}{2} \zeta(2 z)-\frac{1}{4} \frac{\wp^{\prime \prime}(z)}{\wp^{\prime}(z)},
$$

where $\wp(z) \equiv \wp\left(z ; \omega_{1}, \omega_{3}\right)$ and similarly $\zeta(z)$ (see, e.g., [36]).

\section{References}

[1] Haldane F D M, Exact Jastrow-Gutzwiller resonating-valence-bond ground state of the spin-1/2 antiferromagnetic Heisenberg chain with $1 / r^{2}$ exchange, 1988 Phys. Rev. Lett. 60635

[2] Shastry B S, Exact solution of an $S=1 / 2$ Heisenberg antiferromagnetic chain with long-ranged interactions, 1988 Phys. Rev. Lett. 60639

[3] Polychronakos A P, Exact spectrum of $\mathrm{SU}(n)$ spin chain with inverse-square exchange, 1994 Nucl. Phys. B 419553

[4] Haldane F D M, Physics of the ideal semion gas: Spinons and quantum symmetries of the integrable Haldane-Shastry spin chain, in A Okiji and N Kawakami, eds., Correlation Effects in Lowdimensional Electron Systems, Springer Series in Solid-state Sciences, volume 118, 1994, pp. $3-20$

[5] Göhmann F and Wadati M, A note on inverse-square exchange models, 1995 J. Phys. Soc. Jpn. 643585

[6] Basu-Mallick B and Bondyopadhaya N, Exact partition functions of $\mathrm{SU}(m \mid n)$ supersymmetric Haldane-Shastry spin chain, 2006 Nucl. Phys. B $\mathbf{7 5 7} 280$

[7] Gebhard F and Ruckenstein A E, Exact results for a Hubbard chain with long-range hopping, 1992 Phys. Rev. Lett. 68244

[8] Haldane F D M, "Fractional statistics" in arbitrary dimensions: a generalization of the Pauli principle, 1991 Phys. Rev. Lett. 67937

[9] Greiter M and Schuricht D, No attraction between spinons in the Haldane-Shastry model, 2005 Phys. Rev. B $\mathbf{7 1} 224424(4)$

[10] Greiter M, Statistical phases and momentum spacings for one-dimensional anyons, 2009 Phys. Rev. B 79 064409(5)

[11] Haldane F D M, Ha Z N C, Talstra J C, Bernard D and Pasquier V, Yangian symmetry of integrable quantum chains with long-range interactions and a new description of states in conformal field theory, 1992 Phys. Rev. Lett. 692021

[12] Fowler M and Minahan J A, Invariants of the Haldane-Shastry SU(n) chain, 1993 Phys. Rev. Lett. 702325

[13] Haldane F D M, "Spinon gas" description of the $S=\frac{1}{2}$ Heisenberg chain with inverse-square exchange: exact spectrum and thermodynamics, 1991 Phys. Rev. Lett. 661529

[14] Kawakami N, Asymptotic Bethe-ansatz solution of multicomponent quantum systems with $1 / r^{2}$ long-range interaction, 1992 Phys. Rev. B 461005

[15] Ha Z N C and Haldane F D M, Squeezed strings and Yangian symmetry of the Heisenberg chain with long-range interaction, 1993 Phys. Rev. B 4712459

[16] Finkel F and González-López A, Global properties of the spectrum of the Haldane-Shastry spin chain, 2005 Phys. Rev. B 72 174411(6)

[17] Polychronakos A P, Lattice integrable systems of Haldane-Shastry type, 1993 Phys. Rev. Lett. 70 2329

[18] Inozemtsev V I, On the connection between the one-dimensional $S=1 / 2$ Heisenberg chain and Haldane-Shastry model, 1990 J. Stat. Phys. 591143

[19] Whittaker E T and Watson G N, A Course of Modern Analysis (Cambridge University Press) 1927 
[20] Maldacena J M, The large $N$ limit of superconformal field theories and supergravity, 1998 Adv. Theor. Math. Phys. 2231

[21] Witten E, Anti-de Sitter space and holography, 1998 Adv. Theor. Math. Phys. 2253

[22] Gubser S S, Klebanov I R and Polyakov A M, Gauge theory correlators from non-critical string theory, 1998 Phys. Lett. B 428105

[23] Berenstein D, Maldacena J and Nastase H, Strings in flat space and pp waves from $\mathcal{N}=4$ Super Yang Mills, 2002 J. High Energy Phys. 04 013(29)

[24] Minahan J A and Zarembo K, The Bethe-ansatz for $\mathcal{N}=4$ super Yang-Mills, 2003 J. High Energy Phys. 303 013(27)

[25] Serban D and Staudacher M, Planar $\mathcal{N}=4$ gauge theory and the Inozemtsev long range spin chain, 2004 J. High Energy Phys. 06 001(31)

[26] Beisert N, ed., Special Volume: Review on AdS/CFT Integrability, Lett. Math. Phys., volume 99 (Springer) 2012

[27] Inozemtsev V I, Integrable Heisenberg-van Vleck chains with variable range exchange, 2003 Phys. Part. Nucl. 34166

[28] Finkel F and González-López A, A new perspective on the integrability of Inozemtsev's elliptic spin chain, 2014 Ann. Phys.-New York 351797

[29] Basu-Mallick B, Bondyopadhaya N and Sen D, Low energy properties of the $\mathrm{SU}(m \mid n)$ supersymmetric Haldane-Shastry spin chain, 2008 Nucl. Phys. B 795596

[30] Enciso A, Finkel F and González-López A, Spin chains of Haldane-Shastry type and a generalized central limit theorem, 2009 Phys. Rev. E 79 060105(4)

[31] Enciso A, Finkel F and González-López A, Level density of spin chains of Haldane-Shastry type, 2010 Phys. Rev. E 82 051117(6)

[32] Basu-Mallick B and Bondyopadhaya N, Spectral properties of supersymmetric Polychronakos spin chain associated with $A_{N-1}$ root system, 2009 Phys. Lett. A 3732831

[33] Göhmann F and Murakami S, Fermionic representations of integrable lattice systems, 1998 J. Phys. A: Math. Gen. 317729

[34] Inozemtsev V I, The eigenvectors of the Heisenberg Hamiltonian with elliptic form of the exchange spin interaction, 2005 J. Nonlin. Math. Phys. 12395

[35] Sachdev S, Quantum Phase Transitions (Cambridge University Press), second edition 2011

[36] Olver F W J, Lozier D W, Boisvert R F and Clark C W, eds., NIST Handbook of Mathematical Functions (Cambridge Univeristy Press) 2010

[37] Enciso A, Finkel F and González-López A, Thermodynamics of spin chains of Haldane-Shastry type and one-dimensional vertex models, 2012 Ann. Phys.-New York 3272627

[38] Stein E M and Shakarchi R, Complex Analysis (Princeton, N.J.: Princeton University Press) 2003

[39] Barba J C, Finkel F, González-López A and Rodríguez M A, The Berry-Tabor conjecture for spin chains of Haldane-Shastry type, 2008 Europhys. Lett. 83 27005(6)

[40] Barba J C, Finkel F, González-López A and Rodríguez M A, 1/f ${ }^{\alpha}$ noise and integrable systems, 2009 Phys. Rev. E 80 047201(4)

[41] Latorre J I and Riera A, A short review on entanglement in quantum spin systems, 2009 J. Phys. A: Math. Theor. 42 504002(33) 